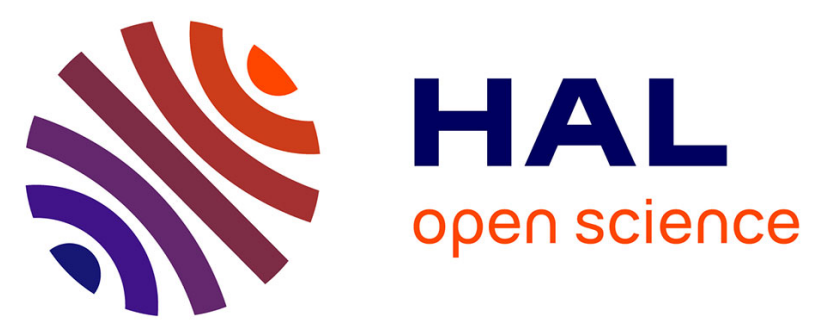

\title{
Etude rhéologique de l'influence de l'ordre et de la transformation ordre- désordre sur le fluage à haute température des solutions solides de surstructure du type B2. (Cu-Zn, Fe-Co, Ag-Mg, Fe3-Al et Ni-Al)
}

P. Delobelle, A. Mermet, C. Oytana

\section{To cite this version:}

P. Delobelle, A. Mermet, C. Oytana. Etude rhéologique de l'influence de l'ordre et de la transformation ordre- désordre sur le fluage à haute température des solutions solides de surstructure du type B2. (Cu-Zn, Fe-Co, Ag-Mg, Fe3-Al et Ni-Al). Revue de Physique Appliquée, 1980, 15 (8), pp.1323-1337. 10.1051/rphysap:019800015080132300 . jpa-00244856

\section{HAL Id: jpa-00244856 https://hal.science/jpa-00244856}

Submitted on 1 Jan 1980

HAL is a multi-disciplinary open access archive for the deposit and dissemination of scientific research documents, whether they are published or not. The documents may come from teaching and research institutions in France or abroad, or from public or private research centers.
L'archive ouverte pluridisciplinaire HAL, est destinée au dépôt et à la diffusion de documents scientifiques de niveau recherche, publiés ou non, émanant des établissements d'enseignement et de recherche français ou étrangers, des laboratoires publics ou privés. 


\title{
Etude rhéologique de l'influence de l'ordre et de la transformation ordre- désordre sur le fluage à haute température des solutions solides de surstructure du type $\mathrm{B}_{2}$. (Cu-Zn, Fe-Co, Ag-Mg, Fe $\mathrm{F}_{3}-\mathrm{Al}$ et $\left.\mathrm{Ni}-\mathrm{Al}\right)$
}

\author{
P. Delobelle, A. Mermet et C. Oytana \\ Laboratoire de Mécanique Appliquée associé au C.N.R.S., \\ Faculté des Sciences et des Techniques de Besançon, Route de Gray, 25030 Besançon Cedex, France \\ (Reçu le 26 février 1980, révisé le 21 avril 1980, accepté le 28 avril 1980)
}

Résumé. - On étudie le fluage des phases polycristallines ordonnées et désordonnées de quelques alliages à surstructure de type $B_{2}$, pour mettre en évidence l'éventuelle influence de l'ordre sur les propriétés rhéologiques de ces matériaux. Les solutions équiatomiques (ou proches) $\mathrm{Cu}-\mathrm{Zn}, \mathrm{Fe}-\mathrm{Co}-2 \mathrm{~V}$ et $\mathrm{Ag}-\mathrm{Mg}$ ont été testées et les résultats de la littérature sur $\mathrm{Ni}-\mathrm{Al}$ et $\mathrm{Fe}_{3}$-Al utilisés.

On utilise une généralisation de l'équation de Dorn $\dot{\varepsilon}_{\mathrm{s}} k T / D G b=C \Phi(S) f(\sigma / G)$, dans laquelle $\Phi(S)$ tient compte $\mathrm{du}$ rôle du degré d'ordre à longue distance $S$. Nous montrons qu'une telle forme est justifiée, l'ordre ne jouant pas sur $f(\sigma / G)$. Si l'on écrit $f(\sigma / G)$ sous la forme classique $\alpha(\sigma / G)^{n}$, on montre que $n$ croît de 3,3 à 10 avec $(\sigma / G)$. Pour expliciter $\dot{\phi}(S)$, on trace pour chacun de ces alliages $M=\operatorname{Ln}\left(\dot{\varepsilon}_{\mathrm{s}} k T / D G b\right)$ à $(\sigma / G)$ constant en fonction de l'inverse de la température. On vérifie que pour les solutions désordonnées on obtient une horizontale. Par contre pour les phases ordonnées, il se produit une brusque variation de $M$ près du point critique $T_{\mathrm{c}}$ lorsque $0 \leqslant S \leqslant 0,6$, cet effet s'estompant progressivement pour $S>0,6$. Les mesures des contraintes internes $\sigma_{\mathrm{i}}$ effectuées sur $\mathrm{Cu}-\mathrm{Zn}$ et $\mathrm{Fe}$-Co présentent un effet analogue, $\sigma_{\mathrm{i}}$ chutant brusquement au voisinage de $T_{\mathrm{c}}$. Un modèle fondé sur des concepts généraux de la physique du fluage à haute température et sur la morphologie des superdislocations dans ce type d'alliage permet de montrer que $\Phi(S)=\exp -\frac{A\left(h, k, l, V_{1}\right) S^{P}}{k T}$.

Pour confronter, sur l'ensemble de ces alliages, les énergies obtenues expérimentalement par la pente des courbes Ln $M=f(1 / T)$ avec les valeurs prévues par ce modèle, on développe $\Phi(S)$ en fonction de la température réduite $T / T_{\mathrm{c}}$, des courbes différentes étant obtenues pour différentes valeurs de $P$. Pour un plan $(h k l)$ considéré, l'ensemble des points se situe sur une même courbe, par exemple pour (110), on trouve $P=2$, valeur très souvent rapportée pour les variations de l'énergie de paroi antiphase avec l'ordre.

La contrainte interne mesurée semble refléter la résistance au mouvement des superdislocations et varie comme une fonction puissance de l'inverse de $\Phi(S)$.

\begin{abstract}
Creep of ordered and disordered polycrystalline solid solutions with a $\mathbf{B}_{2}$ structure is studied to account for the influence of crystallographic order on the rheological properties of these materials. The equiatomic (or close to) solutions $\mathrm{Cu}-\mathrm{Zn}, \mathrm{Fe}-\mathrm{Co}-2 \mathrm{~V}$ and $\mathrm{Ag}-\mathrm{Mg}$ have been tested and the results about $\mathrm{Ni}-\mathrm{Al}$ and $\mathrm{Fe}_{3}-\mathrm{Al}$ gathered from literature have been used. The modified Dorn equation $\dot{\varepsilon}_{\mathrm{s}} k T / D G b=C \Phi(S) f(\sigma / G)$ is used; here, $\Phi(S)$ represents the dependence of creep on the long range order parameter $S$. It is shown that such a relationship is relevant, the crystallographic order having no influence on $f(\sigma / G)$. If $f(\sigma / G)$ takes the classical form $\alpha(\sigma / G)^{n}$, $n$ is shown to increase from 3.3 to 10 with $(\sigma / G)$.

To determine $\Phi(S)$, the curves of $M=\operatorname{Ln}\left(\dot{\varepsilon}_{\mathrm{s}} k T / D G b\right)$ versus $(1 / T)$ with constant $(\sigma / G)$ are drawn for every alloy. It is so checked that for disordered solution an horizontal line is obtained. On the contrary, for the ordered phases, a steep variation of $M$ occurs near the critical temperature $T_{\mathrm{c}}$ when $0 \leqslant S \leqslant 0.6$. This phenomenon vanishing for $S>0.6$. The measurements of the internal stresses $\sigma_{\mathrm{i}}$ performed on $\mathrm{Cu}-\mathrm{Zn}$ and $\mathrm{Fe}$-Co displays a similar effect, $\sigma_{\mathrm{i}}$ falling rapidly in the neighbourhood of $T_{\mathrm{c}}$. A model, lying on general considerations on the physics of creep and on the structure of superdislocations in such alloys leads to $\Phi(S)=\exp -\frac{A\left(h, k, l, V_{1}\right) S^{\boldsymbol{P}}}{k T}$.

To allow the comparison, for the whole set of alloys, between the energies given by the experimental plot of $\operatorname{Ln} M$ versus $(1 / T)$ and those foreseen by this model, $\Phi(S)$ is then written as a function of the reduced temperature $\left(T / T_{\mathrm{c}}\right)$, and so curves can be calculated from the above models for the different values of $P$; it is found that the experimental points fit one of these curve, the $P$ value depending on the $(h k l)$ set of planes considered. For instance to $(110)$ is associated $P=2$, value which has often been reported for the variations of the A.P. boundary energy versus $S$. The internal stress varies as a power function of $\Phi^{\rightarrow 1}(S)$ and seems to represent the opposition to the superdislocation motion.
\end{abstract}


1. Introduction. - L'influence de l'ordre atomique sur les propriétés mécaniques des matériaux et particulièrement des alliages à surstructure $\mathrm{B}_{2}$ (type $\mathrm{Cs}-\mathrm{Cl}$ ) en dessous de la température critique $T_{\mathrm{c}}$, a déjà fait l'objet de nombreux travaux [1,2]. Ainsi, les composés équiatomiques $\mathrm{Cu}-\mathrm{Zn}[3,7], \mathrm{Ag}-\mathrm{Mg}[8,10], \mathrm{Fe}-\mathrm{Co}$ ou $\mathrm{Fe}-\mathrm{Co}-2 \mathrm{~V}$ [11, 16], Ni-Al [17] et $\mathrm{Fe} \simeq 28 \%$ at. $\mathrm{Al}$ [18-19] ont souvent servi de support pour l'étude des différents phénomènes relatifs à l'ordre atomique. Cependant, peu d'études rhéologiques complètes concernent le fluage à haute température de ces alliages $\left(T / T_{\text {Fusion }} \geqslant 0,4\right)$, notamment au voisinage de la transformation ordre-désordre : Cu-Zn [20-25], $\mathrm{Ag}-\mathrm{Mg}$ [26], Fe-Co [27, 28], Ni-Al [29] et Fe-Al [27] $[30,32]$. D'un point de vue purement rhéologique, il ressort de ces travaux que l'influence principale de l'ordre se manifeste sur les paramètres caractéristiques du fluage par un brusque saut au point de transition $T_{\mathrm{c}}[22,25]$. En dessous de $T_{\mathrm{c}}$, lorsque le paramètre d'ordre à longue distance, $S$, croît, son effet semble minime.

Dans le présent article, nous présentons de nouveaux résultats obtenus sur les alliages polycristallins $\mathrm{Cu}-\mathrm{Zn}, \mathrm{Fe}-\mathrm{Co}-2 \mathrm{~V}$ et $\mathrm{Ag}-\mathrm{Mg}$ et nous nous proposons de préciser le rôle de l'ordre sur le fluage à haute température de ces alliages. Pour ce faire, on utilisera une généralisation de l'équation de Dorn [33];

$$
\frac{\dot{\varepsilon}_{\mathrm{s}} k T}{D G b}=C \Phi(S) f\left(\frac{\sigma}{G}\right) .
$$

Dans cette équation, $\dot{\varepsilon}_{\mathrm{s}}$ est la vitesse de fluage stationnaire, $\sigma$ est la contrainte appliquée, $C$ est une constante caractéristique de l'écoulement du matériau, $k T$ et $b$ ont leur signification habituelle, $G$ est le module de Coulomb et $D$ est un coefficient de diffusion dont la détermination sera discutée ultérieurement. La fonction $\Phi$ tient compte du rôle que peut jouer l'ordre à longue distance $S$ par l'intermédiaire de l'énergie de paroi antiphase, par exemple.

Nous reprenons également les différents résultats existants concernant $\mathrm{Ni}-\mathrm{Al}$ et $\mathrm{Fe}-\mathrm{Al}$ et les analysons à l'aide de l'équation (1), en tenant compte des valeurs récentes des différents coefficients de diffusion plau- sibles. De cette manière, et en réalisant la synthèse de l'ensemble de ces résultats, nous tentons d'expliciter globalement la fonction $\Phi(S)$.

2. Méthodes expérimentales. - Les éprouvettes utilisées $(4 \mathrm{~mm}$ de diamètre pour une longueur utile de $25 \mathrm{~mm}$ ) ont été usinées dans des barres obtenues par filage à chaud de lingots dont les compositions pondérales sont données dans le tableau I (alliages équiatomiques). On reporte également les conditions de filage et de recuit d'homogénéisation ultérieurs.

Les essais de fluage ont été réalisés, soit sur une machine type Andrade à contrainte constante pour des allongements supérieurs à $3 \mathrm{~mm}$, soit sur une machine à charge constante pour des valeurs inférieures. L'ensemble thermique de chacune de ces machines est tel qu'il est possible de maintenir une température constante le long de l'échantillon à $\pm 0,5^{\circ} \mathrm{C}$. Les tests ont été généralement poursuivis jusqu'à rupture de l'éprouvette pour les plus fortes contraintes appliquées, la vitesse stationnaire $\dot{\varepsilon}_{\mathrm{s}}$ n'en étant que mieux déterminée, et d'une manière incrémentale pour les contraintes plus faibles. Les très faibles vitesses de fluage ont été mesurées à l'aide d'un transformateur linéaire différentiel.

Bien que sujette à de nombreuses controverses, la détermination des contraintes internes moyennes $\sigma_{\mathrm{i}}$ dans ces matériaux a été réalisée par la méthode de Nix [34] et est donnée par

$$
\sigma_{\mathrm{i}}=\sigma-\sigma^{*} .
$$

La contrainte effective $\sigma^{*}$ est définie comme le décrément de contrainte appliquée qui donne une vitesse de fluage instantanée nulle, cette vitesse étant mesurée juste après le décrément de contrainte. Nous vetrons par la suite, que le paramètre interne $\sigma_{\mathrm{i}}$ déterminé par cette méthode est très sensible au degré d'ordre à longue distance.

Pour chacun de ces alliages, les variations du module de Coulomb avec la température ont été déterminées par des mesures en régime dynamique autour de $300 \mathrm{~Hz}$ par l'intermédiaire d'une éprouvette soumise à des vibrations forcées. $G$ est calculé à partir

\begin{tabular}{|c|c|c|c|}
\hline \multirow{2}{*}{ Alliages } & \multicolumn{3}{|c|}{ Composition pondérale } \\
\hline & $\mathrm{Cu}$ & $\overline{7 n}$ & autres \\
\hline \multirow{4}{*}{$\mathrm{Cu}-\mathrm{Zn}$} & $54,32 \%$ & $45,65 \%$ & autes \\
\hline & $53,27 \%$ & $46,7 \%$ & non \\
\hline & $52 \%$ & $48 \%$ & dosabl \\
\hline & $31,48 \%$ & $48,5 \%$ & \\
\hline Ag-Mg & $\mathrm{Ag}_{0}$ & $\mathrm{Mg}_{0}$ & autres \\
\hline \multirow[b]{2}{*}{$\mathrm{Fe}-\mathrm{Co}-2 \mathrm{~V}$} & \multicolumn{3}{|c|}{ «Supermendur commercial» } \\
\hline & $\begin{aligned} \mathrm{Fe} \\
0 \%\end{aligned}$ & $\begin{aligned} & \text { Co } \\
&\end{aligned}$ & tres \\
\hline
\end{tabular}

Tableau I

Température de filage

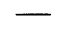

$\simeq 550^{\circ} \mathrm{C}$

$\simeq 630^{\circ} \mathrm{C}$

$\simeq 1150^{\circ} \mathrm{C}$

sous gaine d'acier

\section{Conditions de recuit}

$15 \mathrm{~h}$ à $560^{\circ} \mathrm{C}$ sous argon suivi d'un refroidissement lent

$3 \mathrm{~h}$ à $660^{\circ} \mathrm{C}$ sous argon suivi d'un refroidissement lent

$1 \mathrm{~h}$ à $850^{\circ} \mathrm{C}$ suivi d'un refroidissement lent 
de la fréquence de résonance du montage [35]. Ces fréquences relativement élevées ont été choisies de manière à obtenir une valeur de module non relaxé.

3. Résultats expérimentaux et analyse. - De manière à mettre en évidence l'éventuelle influence de l'ordre sur le fluage, nous utilisons l'équation de Dorn dans sa forme générale (1),

$$
\frac{\dot{\varepsilon}_{\mathrm{s}} k T}{D G b}=C \Phi(S) f\left(\frac{\sigma}{G}\right) .
$$

Celle-ci intègre l'évolution des différentes variables du fluage à haute température, en particulier l'effet de l'ordre sur la diffusion et les constantes élastiques, le terme de structure pouvant être représenté par la fonction $C \Phi(S)$. Donc, si l'on trace $M=\operatorname{Ln}\left(\frac{\dot{\varepsilon}_{\mathrm{s}} k T}{D G b}\right)$ à $(\sigma / G)$ constant en fonction de l'inverse de la température, ce que nous justifierons a posteriori dans l'interprétation, on obtient les variations de $\operatorname{Ln} C \Phi(S)$ avec $1 / T$, donc avec une certaine fonctionnelle de $S$. Pour mieux mettre en évidence le rôle de l'ordre, nous porterons simultanément sur chaque figure et pour chacun des alliages étudiés, l'évolution de $M$ et de $S$ en fonction de $1 / T$. Pour obtenir $M=f(1 / T)$ à $(\sigma / G)$ constant, on trace pour chaque isotherme étudiée la courbe $M=f(\operatorname{Ln} \sigma / G)$, puis on reporte les valeurs de $M$ obtenues pour une verticale donnée c'est-à-dire pour $(\sigma / G)$ fixé.

Cependant, il subsiste un problème quant au choix du coefficient de diffusion $D$ pour ces solutions solides fortement concentrées. En effet, sur ce dernier point, il existe très peu de travaux théoriques, mais il en ressort qu'il subsiste une grande latitude quant au choix de ce coefficient. Pour Brébec et Poirier [36], si l'alliage reste homogène, on doit adopter

$$
D=D^{\prime}=\varphi\left(n_{\mathrm{a}} D_{\mathrm{a}}^{*}+n_{\mathrm{b}} D_{\mathrm{b}}^{*}\right)
$$

et

$$
D=\overline{\bar{D}}=\varphi\left(\frac{D_{\mathrm{a}}^{*} D_{\mathrm{b}}^{*}}{n_{\mathrm{b}} D_{\mathrm{a}}^{*}+n_{\mathrm{a}} D_{\mathrm{b}}^{*}}\right)
$$

dans le cas où un régime stationnaire entre les flux des deux espèces s'établit. Pour Nix et al. [37], si la montée des dislocations contrôle le fluage, on doit avoir

$$
D=\bar{D}=\left(\frac{D_{\mathrm{a}}^{*} D_{\mathrm{b}}^{*}}{n_{\mathrm{b}} D_{\mathrm{a}}^{*}+n_{\mathrm{a}} D_{\mathrm{b}}^{*}}\right),
$$

(formule déjà rapportée par Weertman [38] et Burton et Bastow [39]) alors que le coefficient d'interdiffusion

$$
D=\widetilde{D}=\varphi\left(n_{\mathrm{b}} D_{\mathrm{a}}^{*}+n_{\mathrm{a}} D_{\mathrm{b}}^{*}\right)
$$

est à prendre en compte si le glissement contrôle le fluage. Dans ces formules, $\varphi$ est le facteur thermodynamique

$$
\left[\varphi=\left(1+\frac{\partial \operatorname{Ln} \gamma_{i}}{\partial \operatorname{Ln} n_{i}}\right)\right]
$$

avec $\gamma_{\mathrm{i}}$ l'activité de l'élément $\mathrm{i}$ dans la solution, $n_{\mathrm{a}}$ et $n_{\mathrm{b}}$ sont les fractions atomiques des constituants de l'alliage et $D_{\mathrm{a}}^{*}, D_{\mathrm{b}}^{*}$ respectivement leur coefficient d'autodiffusion dans l'alliage. Notons que pour des solutions équiatomiques $D^{\prime}=\widetilde{D}$. Il est difficile de choisir d'un point de vue purement théorique l'un de ces quatre coefficients. Pour la suite des calculs nous adopterons la formulation de Nix et al. puisqu'elle semble avoir reçu une confirmation expérimentale pour des solutions solides d'Al-Zn fortement concentrées [37]. De plus, comme nous le verrons cidessous, les énergies d'activation de $D_{\mathrm{a}}^{*}$ et $D_{\mathrm{b}}^{*}$ étant très proches pour ces alliages, les différentes formules précédentes conduisent, en faisant abstraction du coefficient $\varphi$, à des énergies de diffusion apparentes très proches. Par contre le terme

$$
Q_{\varphi}=\left(\frac{\partial \operatorname{Ln} \varphi}{\partial-\frac{1}{k T}}\right)
$$

peut prendre des valeurs non négligeables. On peut noter que pour la phase $\beta$ de l'alliage équiatomique Ag-Zn, $D_{\mathrm{Ag}}^{*}$ étant assez différent de $D_{\mathrm{Zn}}^{*}$, il est possible de lever expérimentalement l'indétermination et de montrer que $\bar{D}$ ou $\bar{D}$ sont les deux seuls coefficients qui conduisent à une analyse cohérente [35], [67].

Pour simplifier l'exposé des nombreux résultats. nous présentons successivement les différents alliages en insistant sur ce qui leur est intrinsèque. Le tableau II constitue l'index bibliographique et résume les caractéristiques obtenues compte tenu des conditions expérimentales.

\subsection{Alliages $\mathrm{Cu}-\mathrm{Zn}$ PROCHES DE la COMPOSITION} ÉQUIATOMIQUE. - Pour l'ensemble de ces solutions et compte tenu du domaine de contraintes exploré, la fonction $f(\sigma / G)$ prend la forme $(\sigma / G)^{n}$ avec $n \simeq 3,3$ de part et d'autre de $T_{\mathrm{c}}$. Cette valeur est en parfait accord avec celle obtenue par Brown et Lenton [22] pour des monocristaux.

i) Cas de l'alliage à $47,3 \%$ at. de $\mathrm{Zn}$. - On reporte sur la figure 1 les courbes $M=f(1 / T)$ pour deux valeurs de $(\sigma / G)$ et pour $\bar{D}$ et $\widetilde{D}$ calculés respectivement à partir des travaux de Kuper et al. [40] et Ugaste et al. [41]. Les valeurs expérimentales de $S$ sont celles rapportées par Rathmann et Als. Nielsen [42] à partir de mesures de diffraction neutroniques et sont comparées aux courbes déduites des théories de BraggWilliams (B. W.) et de Cowley (C.) en ne considérant que les premiers voisins;

$$
\begin{array}{ll}
\text { B. W. } ; & 2 S \frac{T_{\mathrm{c}}}{T}=\operatorname{Ln}\left(\frac{1+S}{1-S}\right) \\
\text { C. } ; & 2 S^{2} \frac{T_{\mathrm{c}}}{T}=\operatorname{Ln}\left(\frac{1+S^{2}}{1-S^{2}}\right) .
\end{array}
$$

L'accord avec la théorie de Cowley est assez bon : nous reviendrons ultérieurement sur ce point. 


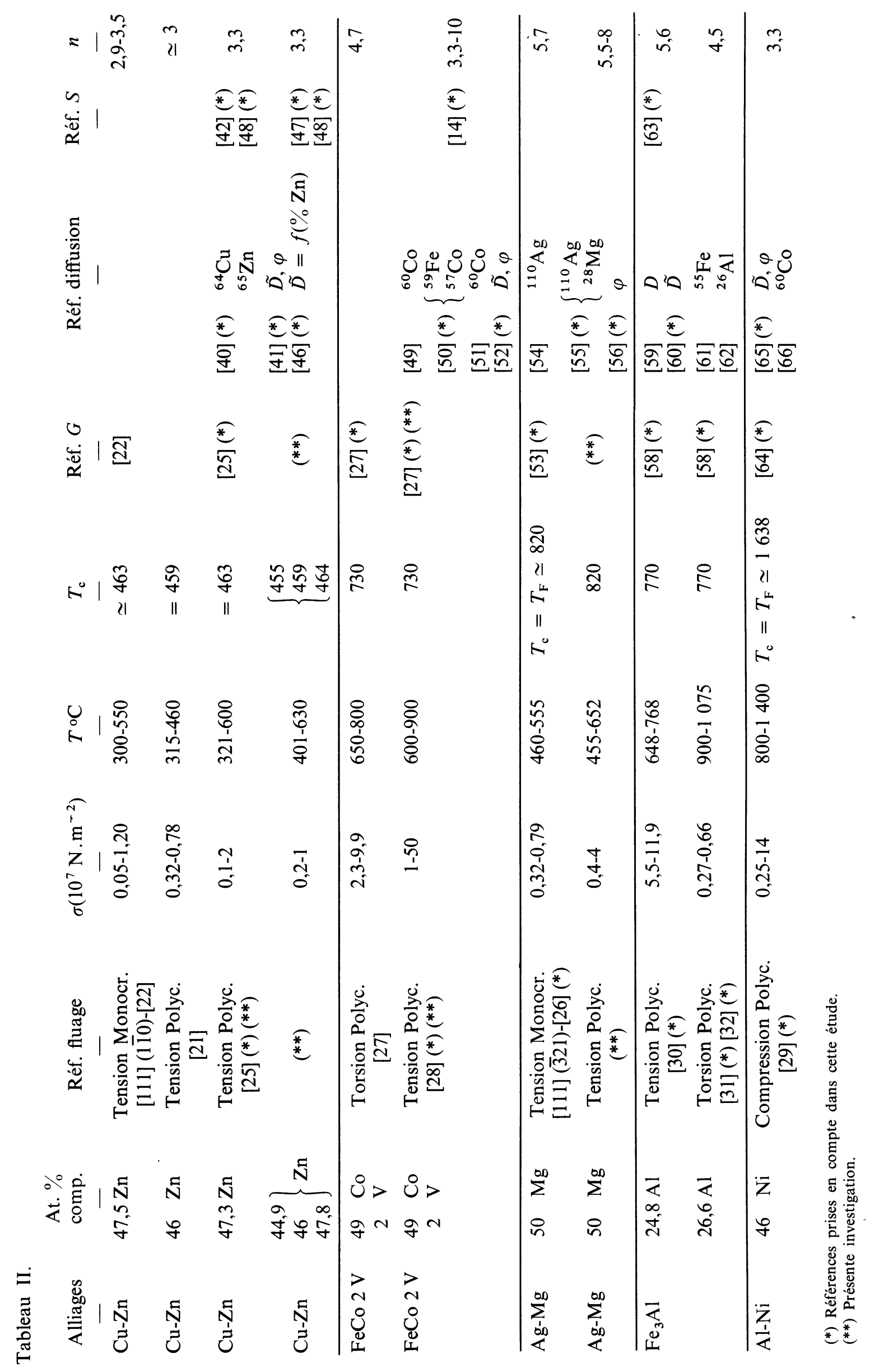




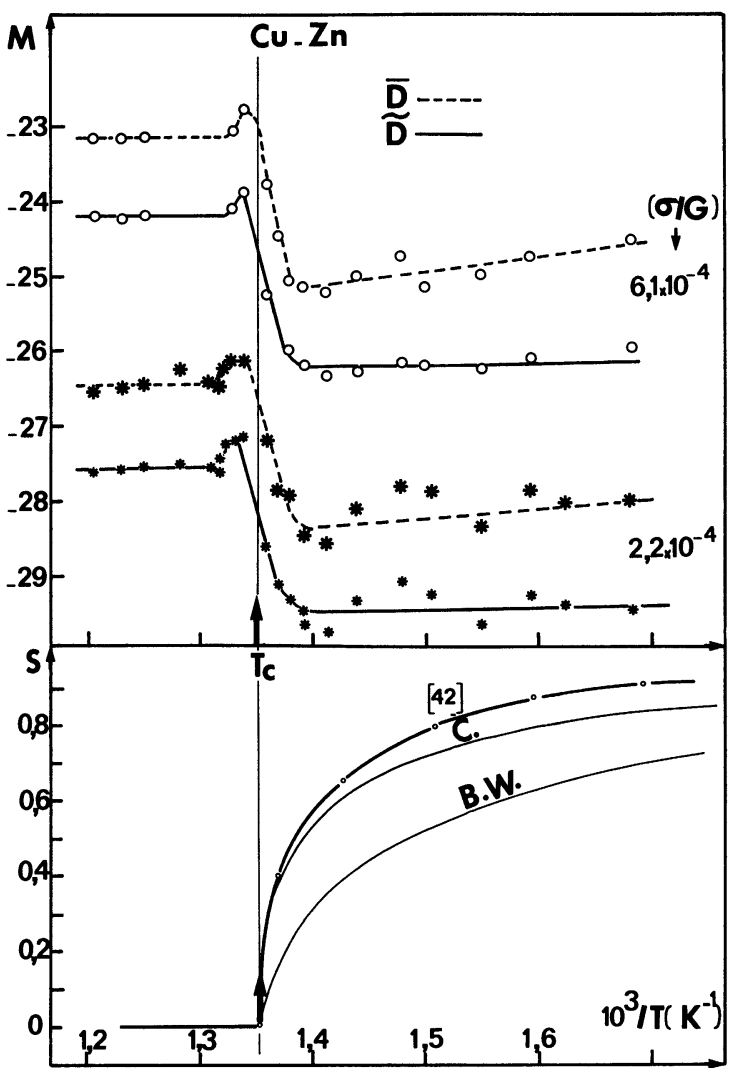

Fig. 1. - Alliage $\mathrm{Cu}-\mathrm{Zn}$ équiatomique. Evolution de $M$ et de $S$ avec $(1 / T)$ de part et d'autre de $T_{\mathrm{c}}$.

[Equiatomic Cu-Zn alloy. $M$ and $S$ evolutions with $1 / T$ on each side of $T_{\mathrm{c}}$.]

Cette figure appelle à de nombreuses remarques : - L'influence du choix du coefficient de diffusion par l'intermédiaire du facteur thermodynamique $\varphi$ est très nette. Non seulement il translate $M$, mais il joue sur la pente des courbes par l'intermédiaire de l'énergie $Q_{\varphi}$ qui est de l'ordre de $-0,11 \mathrm{eV}$ pour la phase $\beta^{\prime}$ partiellement ordonnée alors qu'elle est négligeable pour la phase $\beta$ désordonnée [41]. Le coefficient $\tilde{D}$ semble le plus plausible pour plusieurs raisons : d'une part parce que si l'on prend $\bar{D}$ on ne voit pas très bien physiquement pourquoi $M=f(1 / T)$ présenterait un minimum pour $S \simeq 0,5$ alors que l'ordre croît continûment et d'autre part la valeur de $n \simeq 3$, en accord avec les différentes théories du fluage par glissement visqueux des dislocations [43], du moins pour les plus faibles contraintes et si l'on accrédite ces théories à l'aide de la seule valeur de $n$ (Fig. 10 et $\S 3.6$ ), est en désaccord avec $\bar{D}$ compte tenu des hypothèses de Nix et al. [37] pour le choix de $D$.

- Alors que $M$ est constant pour la phase désordonnée $(S=0)$, il se produit une assez brusque transition au voisinage de $T_{\mathrm{c}}$. Cependant, celle-ci n'est pas ponctuelle mais se produit sur un certain invervalle de température $(0 \leqslant S \leqslant 0,6)$, contrairement à ce que rapportent Brown et al. [22]. Pour des températures inférieures $(S>0,6)$, l'ordre semble ne plus avoir d'effet important. Ainsi, la forte concavité der courbes d'Arrhénius $\operatorname{Ln} \dot{\varepsilon}_{\mathrm{s}}=f(1 / T)_{\sigma}[21,22.25]$ est complètement intégrée sitôt que l'on s'éloigne de $T_{\mathrm{c}}$ par le coefficient de diffusion qui présente une courbure analogue. Ce phénomène est dû au mécanisme de diffusion à six bonds ayant lieu dans la structure Cs-Cl [44].

Sur la figure 2, on reporte pour plusieurs contraintes l'évolution de $\sigma_{\mathrm{i}}=f\left(\frac{1}{T}\right)$. Bien que ces valeurs soient très faibles et imprécises compte tenu de la technique expérimentale et des différents problèmes qui s'y rattachent $[24,25,45]$, il existe néanmoins une transition bien marquée au voisinage de $T_{\mathrm{c}}$. Sa signification sera discutée dans le paragraphe 4 .

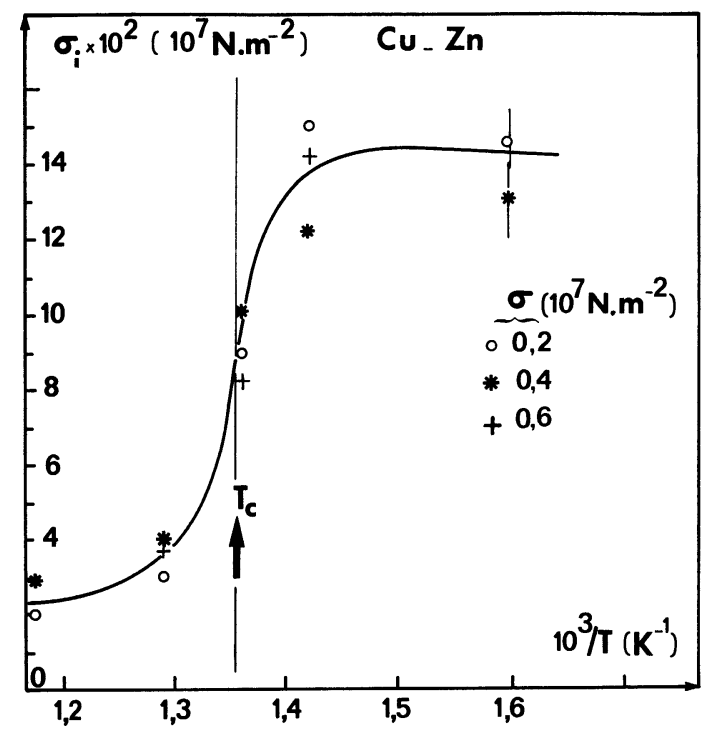

Fig. 2. - Variation de $\sigma_{\mathrm{i}}=f(1 / T)$ de part et d'autre de $T_{\mathrm{c}}$, pour plusieurs niveaux de contraintes appliquées.

[ $\sigma_{\mathrm{i}}$ versus $1 / T$ on each side of $T_{\mathrm{c}}$ for several applied stress levels.]

ii) Etude en fonction de la composition. - Pour confirmer l'existence de cette transition et compte tenu des mesures récentes de $\widetilde{D}$ en fonction du pourcentage de zinc [46], nous avons réalisé des essais de fluage en fonction de la concentration, de façon à franchir le point critique d'une manière isotherme. On diminue ainsi l'incertitude sur $\widetilde{D}$ au voisinage de $T_{\mathrm{c}}$.

Sur la figure 3, on reporte

$$
C \Phi(S)=\left(\frac{\dot{\varepsilon}_{\mathrm{s}} k T}{\widetilde{D} G b}\right) /\left(\frac{\sigma}{G}\right)^{3,3}=f(\% \mathrm{Zn})
$$

pour différentes isothermes et deux valeurs de $(\sigma / G)$. Les variations de $S$ avec la concentration pour chaque isotherme au voisinage de $T_{\mathrm{c}}$, ont été calculées à l'aide des résultats de Walker et Chipman [47]. En effet, à partir de la théorie de Cowley, qui approxime assez bien la réalité, ces auteurs montrent que lorsque 


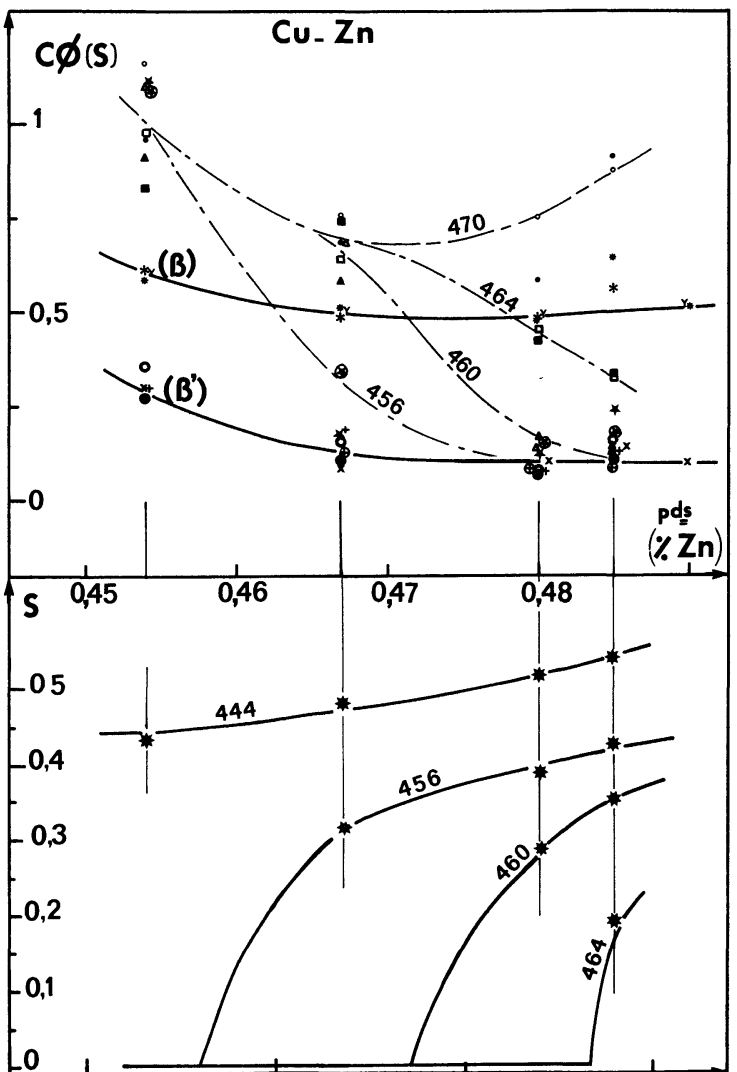

Fig. 3. - Variation de $C \Phi(S)$ et de $S$ avec la concentration en zinc pour différentes isothermes.

$(\sigma / G)$

\begin{tabular}{|c|c|c|c|c|}
\hline & & gende $T^{\circ} \mathrm{C}$ & $1,23 \times 10^{-4}$ & $3,35 \times 10^{-4}$ \\
\hline & & - & - & - \\
\hline & 604 & - & * & \\
\hline & 573 & - & $\mathbf{Y}$ & \\
\hline$\beta$ & 522 & - & * & \\
\hline & 470 & $\cdots$ & $\bullet$ & $\circ$ \\
\hline Transition & 464 & $\cdots$ & घ & $\square$ \\
\hline$\beta \rightarrow \beta^{\prime}$ & 460 & $-\cdots$ & $\Delta$ & $\Delta$ \\
\hline & $(456$ & $\cdots$ & $\circledast$ & $\star$ \\
\hline & $\int 444$ & - & $x$ & \\
\hline$\beta^{\prime}$ & 431 & - & $\bullet$ & O \\
\hline & $(401$ & - & $\oplus$ & + \\
\hline
\end{tabular}

[Variations of $C \Phi(S)$ and $S$ versus the zinc content for different isothermic curves.]

$T \rightarrow T_{\mathrm{c}} . S$ varie avec la composition atomique de cuivre, $\% \mathrm{Cu}=\frac{1}{2}(1+\varepsilon)$, comme

$$
S=-\frac{3^{1 / 4}}{\left(1+3 \varepsilon^{2}\right)^{1 / 2}}\left(\frac{T_{\mathrm{c}}}{T}-1\right)^{1 / 4} .
$$

Entre les phases ordonnée et désordonnée $\left(\beta^{\prime}\right.$ et $\left.\beta\right)$ il subsiste bien une différence de niveau sur $C \Phi(S)$. Pour 456,460 et $464{ }^{\circ} \mathrm{C}$ on franchit la transition de manière isotherme et les variations de $C \Phi(S)$ sont qualitativement en accord avec celles de $S$. On confirme donc l'existence d'une variation progressive lorsque $S$ chute approximativement de 0,5 à zéro.

Comme sur la figure 1, on remarque qu'il se produit un léger adoucissement juste au-dessus de $T_{\mathrm{c}}$ (voir l'isotherme $470^{\circ} \mathrm{C}$ par exemple), suivi d'une faible consolidation. Ce phénomène a déjà été signalé par Oytana et al. [23] et semble associé d'une part à la disparition de l'ordre à courte distance qui subsiste jusqu'à $T \simeq T_{\mathrm{c}}+80^{\circ} \mathrm{C}$ [48] et d'autre part à une recristallisation très nette juste au-dessus du point critique.

Pour $T=460^{\circ} \mathrm{C}$, on reporte sur la figure 4 l'évolution de $\sigma_{\mathrm{i}}$ avec la concentration et l'on confirme ainsi l'existence d'un saut au voisinage de $T_{\mathrm{c}}$.

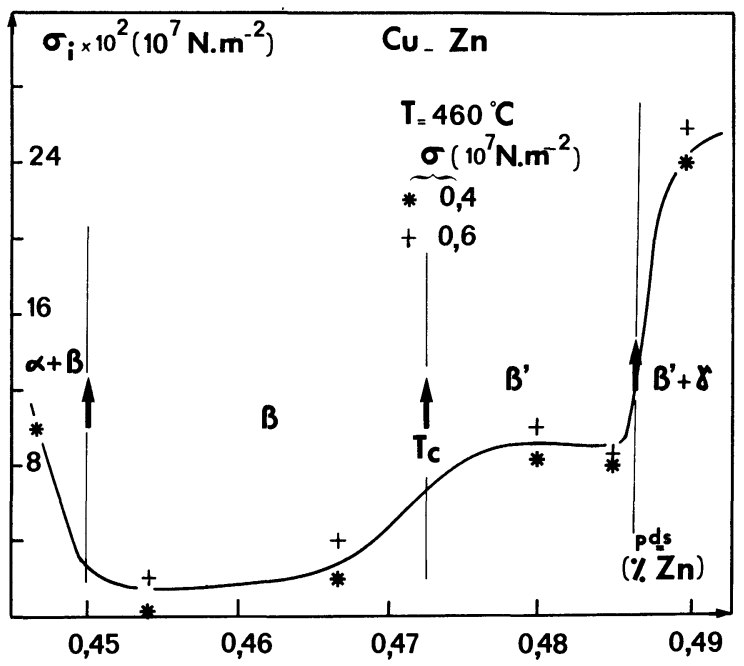

Fig. 4. - Evolution de $\sigma_{\mathrm{i}}=f(\% \mathrm{Zn})$ pour $T=460^{\circ} \mathrm{C}$ et deux niveaux de contrainte appliquée.

[Evolution of $\sigma_{\mathrm{i}}$ with the $\mathrm{Zn}$ percentage at $T=460^{\circ} \mathrm{C}$ for two applied stress levels.]

3.2 Alliage Fe-Co-2 V. - Il faut noter que cet alliage ternaire $(2 \% \mathrm{~V})$ a été choisi pour sa bonne ductilité compatible avec l'étude proposée, contrairement à l'alliage binaire $\mathrm{Fe}$-Co équiatomique qui est extrêmement fragile et dont la faible déformation est essentiellement intergranulaire [13, 14, 28].

Cet alliage a été étudié sur un large domaine de contraintes et de températures, contrairement à l'étude de Hren [27] (Tableau II), et l'on peut montrer que si, dans (1) on prend $f(\sigma / G)=\alpha(\sigma / G)^{n}, n$ croît de 3,3 pour les faibles contraintes à $n \simeq 10$ pour les contraintes les plus élevées, aussi bien pour la phase $\alpha$ désordonnée que pour la phase $\alpha^{\prime}$ partiellement ordonnée.

Pour le choix de $D$, nous raisonnerons dans le cadre d'un alliage binaire, ce qui semble parfaitement justifié compte tenu de l'identité entre l'énergie d'activation apparente obtenue en fluage et celle de la diffusion moyenne, assez loin de la transformation [28]. Il existe de nombreuses mesures de diffusion pour l'alliage désordonné [49-51], alors que les seules valeurs disponibles dans l'état ordonné sont dues à Fishman et al. [50]. Au regard de la très faible différence existant entre $D_{{ }_{59 \mathrm{Fe}}}^{*}$ et $D_{60 \mathrm{Co}}^{*}$, les formules (5) et (6) conduisent au même résultat au terme $\varphi$ près. Pour la phase $\alpha, \varphi$ est proche de l'unité $\left(0,77\right.$ à $800^{\circ} \mathrm{C}$ par 
exemple) alors que $Q_{\varphi} \simeq 0,2 \mathrm{eV}$ [52]. En dessous de $T_{\mathrm{c}}$ aucune valeur de $\varphi$ n'est disponible dans la littérature. Pour des raisons analogues à celles citées précédemment, nous prendrons donc pour la suite de l'étude, $\tilde{D}$ pour la phase $\alpha$ et une valeur moyenne de $\widetilde{D}$ avec $\varphi=1$ pour la phase $\alpha^{\prime}$

Comme dans le cas des laitons, alors que $M$ est constant pour la phase désordonnée (Fig. 5), il se

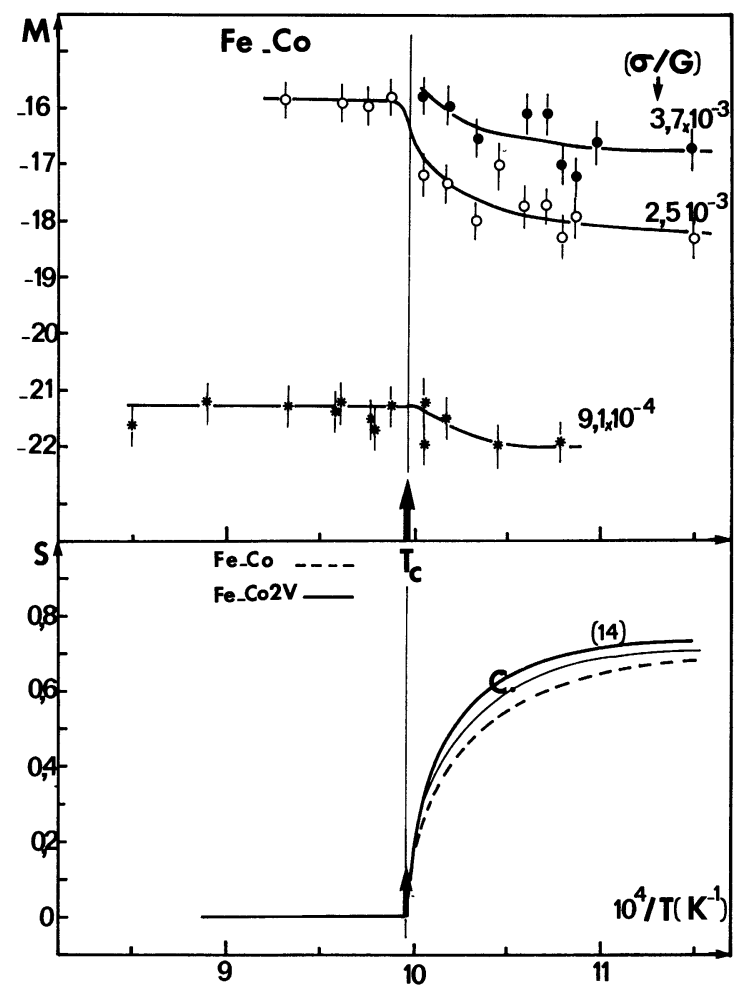

Fig. 5. - Alliage Fe-Co-2 V ; Evolution de $M$ et de $S$ avec $(1 / T)$ de part et d'autre de $T_{\mathrm{c}}$ pour différentes valeurs de $(\sigma / G)$.

[Fe-Co-2 $\mathrm{V}$ alloy, $M$ and $S$ versus $1 / T$ on each side of $T_{\mathrm{c}}$ for different $\sigma / G$ values.]

produit une brusque variation (consolidation) au voisinage de $T_{\mathrm{c}}=730^{\circ} \mathrm{C}$, qui s'estompe lorsque $S$ croît. Les valeurs de $S$ sont celles mesurées par Demenet et al. [14]. L'amplitude du phénomène s'estompe lorsque $(\sigma / G)$ devient faible, ce qu'on peut interpréter comme une augmentation de la taille des sous-cellules de structure qui tend vers les dimensions du diamètre des grains très fins de cet alliage $[13,28]$.

- L'allure de $\sigma_{\mathrm{i}}=f(1 / T)$ pour différents niveaux de contrainte est donné sur la figure 6 . Comme précédemment, alors que son niveau est quasiment nul pour la phase $\alpha$, il croît cette fois-ci de manière considérable à partir de $T_{\mathrm{c}}$. L'interprétation sera donnée dans le paragraphe 4 .

3.3 Solution ÉQuiatomique Ag-Mg. - Cette solution solide équiatomique présente un état ordonné jusqu'à son point de fusion $T_{\mathrm{F}} \simeq 820^{\circ} \mathrm{C}$ [8]. Mukherjee et Dorn [26] ont étudié le fluage de monocristaux selon [111] $(\overline{3} 21)$, pour des contraintes comprises entre 0,3 et $0,8 \times 10^{7} \mathrm{~N} . \mathrm{m}^{-2}$, et obtiennent

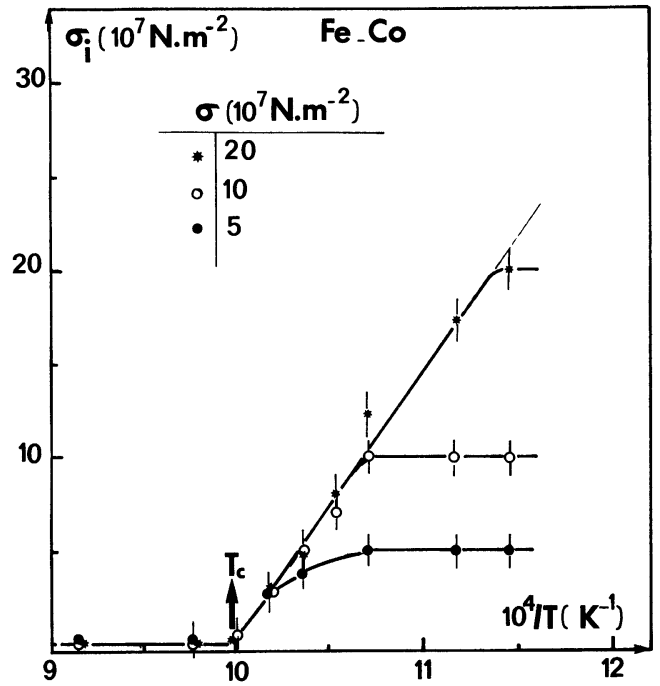

Fig. 6. - Représentation de $\sigma_{\mathrm{i}}=f(1 / T)$ pour différentes contraintes appliquées.

$\left[\sigma_{\mathrm{i}}=f(1 / T)\right.$ plots for different applied stresses. $]$

un exposant $n$ de l'ordre de 5,7. Nous reprenons cette étude dans le cas de polycristaux sur une gamme plus étendue de température et de contrainte et l'on montre ainsi que $n$ croît de 5,5 à 8 avec $(\sigma / G)$.

Nous avons calculé $M$ pour les mono et polycristaux en prenant les valeurs récentes des coefficients de diffusion des radiotraceurs rapportés par Domian et Aaronson [55], les valeurs de $G$ mesurées étant un peu plus faibles que celles déduites de $C_{11}$ en considérant le matériau comme isotrope $\left(G \simeq 0,4 C_{11}\right)$ [53]. Comme précédemment $\bar{D}$ et $\tilde{D}$ sont très voisins au paramètre $\varphi$ près qui translate l'ensemble des courbes d'une valeur constante ; $\varphi \simeq 6,6$ et $Q_{\varphi} \simeq 0,04 \mathrm{eV}$ [56]. Sur la figure 7 on reporte $M=f(1 / T)$ calculé avec $D$ pour les mono et polycristaux et l'on constate que $M$ est pratiquement indépendant de $S$ pour la plage $0,8 \leqslant S \leqslant 0,95$, valeurs estimées à l'aide de la théorie de Cowley, compte tenu du manque de résultats expérimentaux.

Les mesures de contraintes internes sont très imprécises mais tendent à montrer qu'il existe un niveau moyen non nul, fonction de la contrainte, et qui croît avec $(1 / T)$.

3.4 CAS DE L'Alliage $\mathrm{Fe}_{3} \mathrm{Al}$. - L'alliage $\mathrm{Fe}_{3} \mathrm{Al}$ a beaucoup été étudié du point de vue structural car il possède deux types de structures ordonnées, à savoir, $\mathrm{DO}_{3}$ aux plus faibles températures et $\mathrm{B}_{2}$ pour les températures intermédiaires, puis devient désordonné jusqu'à son point de fusion [57]. Nous nous intéressons présentement à la transition ordonnée $\mathrm{B}_{2} \rightarrow$ désordonnée, $T_{\mathrm{c}}$ se trouvant vers $770^{\circ} \mathrm{C}$. On peut noter que l'alliage équiatomique $\mathrm{FeAl}$ a très peu été étudié à cause des difficultés de son élaboration et de son extrême fragilité. D'autre part, il est ordonné $B_{2}$ jusqu'à son point de fusion.

Les résultats concernant le fluage de cet alliage sont peu nombreux et disparates, ainsi ceux de la phase 


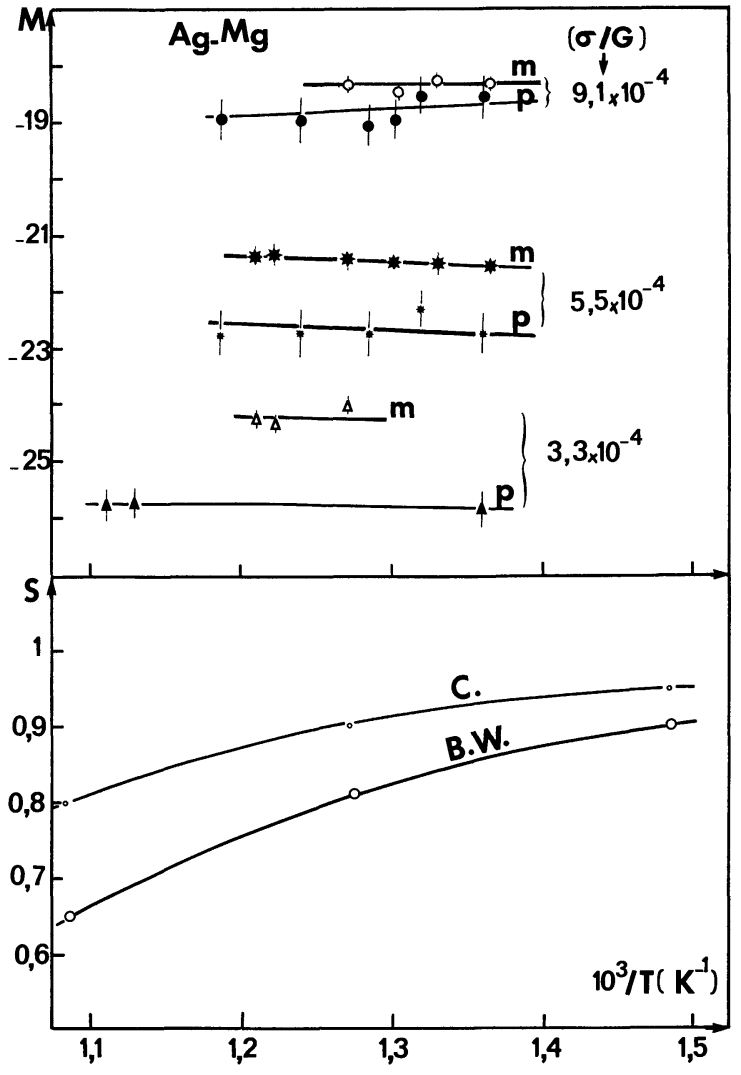

Fig. 7. - Alliage $\mathrm{Ag}-\mathrm{Mg}$ équiatomique; Evolution de $M$ et de $S$ avec $(1 / T)$ pour différents $(\sigma / G)$ dans le cas de mono et de polycristaux.

[Equiatomic Ag-Mg alloy; $M$ and $S$ variation with $1 / T$ for different $\sigma / G$ values in the single and the polycrystal cases.]

ordonnée sont dus à Lawley et al. [30], alors que ceux de la phase désordonnée sont dus à Surova et al. [31]. Pour confronter ces résultats, nous avons converti les vitesses et les contraintes de torsion des expériences de Surova et al. en contraintes et vitesses de traction équivalente en supposant la validité du critère de Von Misès. Ainsi $\bar{\sigma}=\sqrt{3} \tau$ et $\dot{\varepsilon}_{\mathrm{s}}=\dot{\Gamma}_{\mathrm{s}} / \sqrt{3}$. Le module de Coulomb de ce composé a été déterminé par Hren [58]. Il existe quelques mesures de diffusion concernant cette solution [59-62], mais il s'avère qu'il subsiste une très grande dispersion des résultats pour la phase ordonnée, contrairement à la phase haute température où les points convergent de manière significative [59-61]. Cependant, il ne fait aucun doute qu'il existe un rapport important entre $D_{\mathrm{Al}}^{*} / D_{\mathrm{Fe}}^{*} \simeq 6$ dans la phase ordonnée. Compte tenu de ce manque d'information nous prendrons les résultats cohérents sur $\tilde{D}$ rapportés par Hirano et al. [60] que nous extrapolerons vers les basses températures. Les valeurs de $S$ sont celles rapportées par Khachaturyan [63].

Les courbes $M=f(1 / T)$ sont ainsi tracées sur la figure 8 et l'on constate qu'il semble exister une transition près de $T_{\mathrm{c}}$, si l'on extrapole les résultats des hautes températures pour des valeurs de $(\sigma / G)$ compatibles avec celles relatives à la phase ordonnée (trait pointillé). $M$ décroît ensuite légèrement avec $S$.

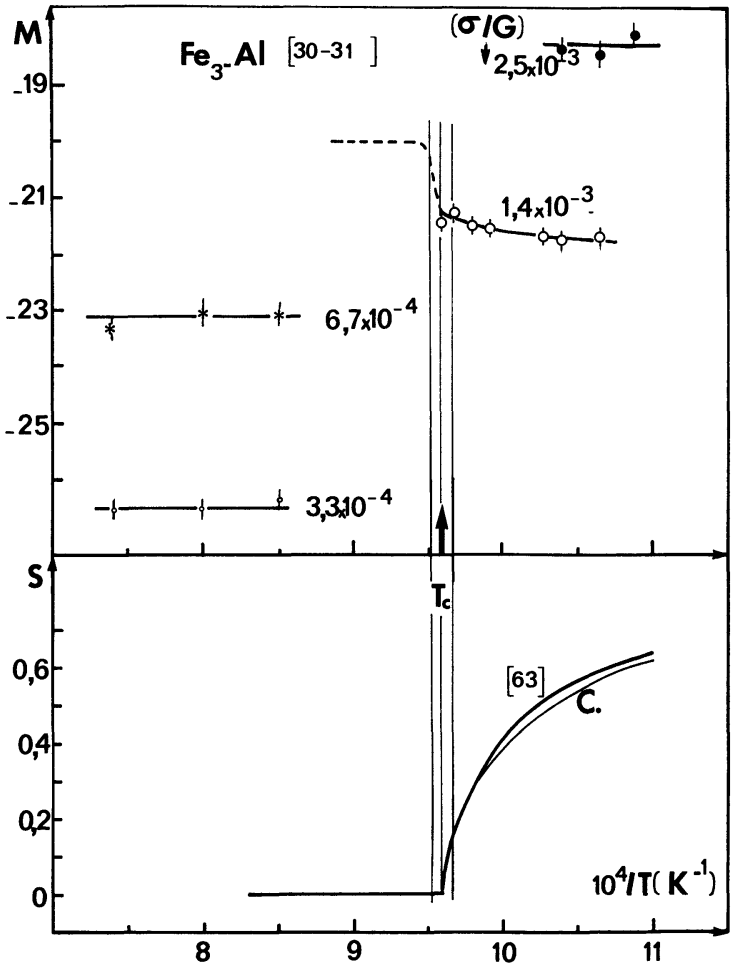

Fig. 8. - Alliage $\mathrm{Fe}_{3} \mathrm{Al}$. On représente $M$ et $S$ en fonction de $(1 / T)$ lors de la transformation ordonné $\mathrm{B}_{2} \rightarrow$ désordonné.

$\left[\mathrm{Fe}_{3} \mathrm{Al}\right.$ alloy. $M$ and $S$ versus $1 / T$ are plotted during the transition ordered $\mathbf{B}_{2} \rightarrow$ disordered.]

Bien que cette représentation soit assez incertaine compte tenu des différentes extrapolations effectuées, la transition obtenue près de $T_{\mathrm{c}}$ est conforme aux observations de Hren [27] concernant cet alliage.

3.5 CAS DE LA SOlution Ni-Al. - Comme le composé $\mathrm{Ag}-\mathrm{Mg}$ étudié précédemment, la solution $\mathrm{Ni}-\mathrm{Al}$ équiatomique (ou voisine) présente un état ordonné jusqu'à son point de fusion, $T_{\mathrm{F}} \simeq 1638^{\circ} \mathrm{C}$ [29]. Vandervoort et al. [29] ont étudié à l'aide d'essais de compression le fluage de la solution $\mathrm{Al}-46 \%$ at. $\mathrm{Ni}$ pour des températures assez proches de $T_{\mathrm{F}}$ et trouvent un exposant $n \simeq 3,3$. Ils donnent également le comportement des solutions à 49,6 et $56 \%$ at. $\mathrm{Ni}$.

Nous avons calculé $M$ à l'aide des valeurs de $G$ déduites des coefficients élastiques mesurés par Wasilewski [64] et de $\tilde{D}=f(\% \mathrm{Ni})$ très récemment étudié par Shankar et Seigle [65]. On peut noter que les anciens travaux de Berkowitz et al. [66] sur la diffusion de ${ }^{60} \mathrm{Co}$ dans $\mathrm{Ni}-\mathrm{Al}$ sont cohérents avec ceux de Sankar et al. [65] qui montrent d'ailleurs que $\varphi \simeq 20$ pour $\mathrm{Al} 46 \% \mathrm{Ni}$. Au vu de la valeur expérimentale de $n \simeq 3$, on justifie le choix de $\widetilde{D}$, du moins en faisant abstraction des restrictions énumérées précédemment (3.1.i).

Sur la figure 9 on remarque que $M$ décroît légèrement avec $(1 / T)$ alors que $0,7 \leqslant S \leqslant 0,95$ (valeurs estimées à l'aide de la formule de Cowley (10)).

3.6 CONCLUSIONS LIÉES AUX FAITS EXPÉRIMENTAUX. - En conclusion, d'un point de vue rhéologique, 


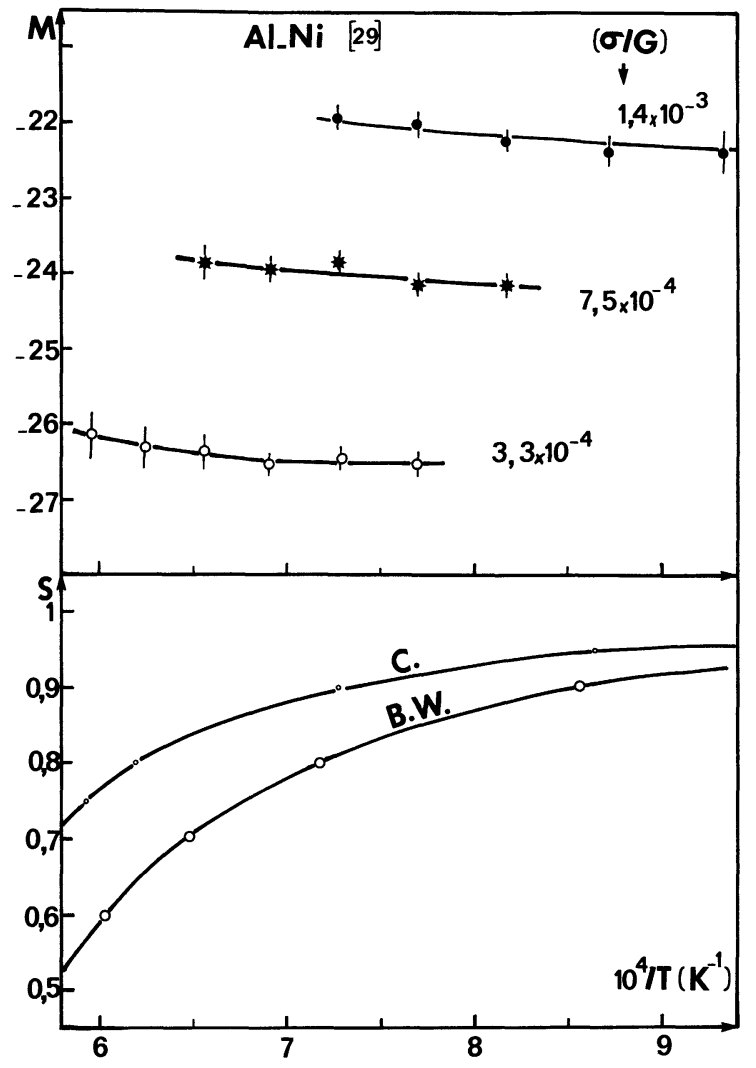

Fig. 9. - Alliage Al-Ni équiatomique. Evolution de $M$ et $S$ avec $(1 / T)$ près du point de fusion.

[Equiatomic Ni-Al alloy. $M$ and $S$ variations with $1 / T$ in the melting temperature neighbourhood.]

nous pouvons dire que la résistancè au fluage est une fonction notablement décroissante de $S$ au voisinage de $T_{\mathrm{c}}$ lorsque $0 \leqslant S \leqslant 0,6$ et que cet effet s'estompe progressivement lorsque $S$ croît ensuite vers l'unité. L'ordre à courte distance ne semble avoir d'effet qu'immédiatement au-dessus de $T_{\mathrm{c}}, M$ étant très vite constant au-delà.

D'autre part, sur la figure 10 , nous rassemblons sur un diagramme classique de Dorn,

$$
\operatorname{Ln} \frac{\dot{\varepsilon}_{\mathrm{s}} k T}{D G b}=f\left(\operatorname{Ln} \frac{\sigma}{G}\right)
$$

l'ensemble des résultats obtenus dans cette étude. Evidemment les droites relatives aux phases ordonnées représentent le tracé moyen obtenu pour $S$ suffisamment grand. Nous reportons en plus les droites calculées par Dorn [33] à partir des études de Brown et Lenton [22] pour des monocristaux de $\mathrm{Cu}-\mathrm{Zn}$ (courbes $5^{\prime}$ ). On s'aperçoit que ce réseau se situe dans la même région du diagramme ce qui est assez satisfaisant compte tenu de l'identité des structures [67]: ainsi par exemple pour

$$
T / T_{\mathrm{F}} \simeq 0,6 \text { et }(\sigma / G) \simeq 3,3 \times 10^{-4}
$$

on obtient des vitesses aussi différentes que $10^{-2} \mathrm{~s}^{-1}$ pour $\mathrm{Cu}-\mathrm{Zn}$ et $8 \times 10^{-6} \mathrm{~s}^{-1}$ pour $\mathrm{FeCoV}$ mais des points voisins sur la figure 10 .

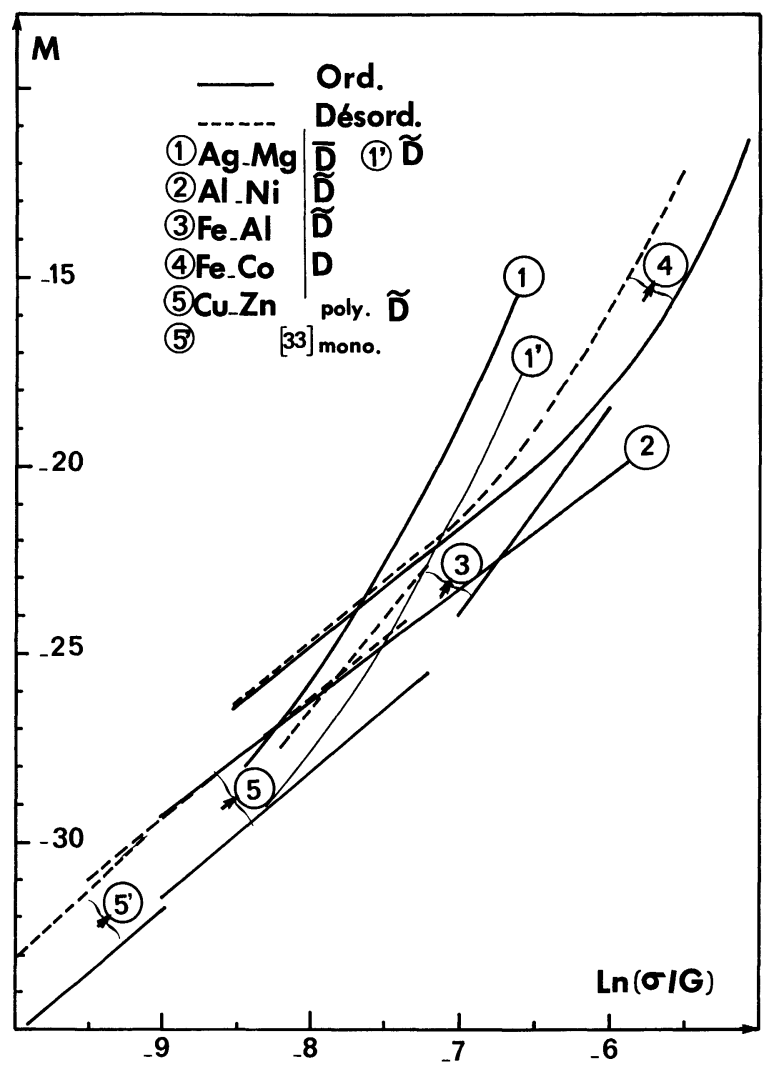

Fig. 10. - Représentation en coordonnées réduites de Dorn $\operatorname{Ln} \frac{\dot{\varepsilon}_{\mathrm{s}} k T}{D G b}=f\left(\operatorname{Ln} \frac{\sigma}{G}\right)$ de l'ensemble des résultats obtenus.

[Dorn's reduced coordinates $\operatorname{Ln} \frac{\dot{\varepsilon}_{\mathrm{s}} k T}{D G b}=f\left(\operatorname{Ln} \frac{\sigma}{G}\right)$ representation of the whole set of available results.]

Si l'on écrit $f(\sigma / G)=\alpha(\sigma / G)^{n}, n$ a tendance à croître avec $(\sigma / G)$ de 3,3 vers des valeurs typiquement de l'ordre de 10. Une loi plus générale, type Garofalo par exemple [68], en [sinh $\alpha(\sigma / G)]^{n}$, serait plus apte à décrire l'ensemble des phénomènes. Un autre fait important est qu'en général, pour un alliage donné, les courbes relatives aux états ordonné et désordonné restent parallèles, ce qui montre que l'exposant $n$ ne subit pratiquement aucune variation lors de la transition. On montre ainsi que $n$ n'est pas fonction de $S$ et que l'ordre se manifeste bien par une fonction $\Phi(S)$.

Donc, si l'on attribue, comme on a l'habitude de le faire, un mécanisme de déformation à chaque valeur de $n$, on doit avoir le même mécanisme de part et d'autre de $T_{\mathrm{c}}$. Il faut remarquer tout de même que l'identification à l'aide de la seule valeur de $n$ est assez abusive compte tenu de son évolution avec $(\sigma / G)$. Dans cette étude, seul $n \simeq 3,3$ pour les plus faibles contraintes semble significatif. 
4. Interprétation et discussion. Influence de l'ordre. A l'aide d'un modèle simple basé sur des concepts généraux, nous allons expliciter la fonction $\Phi(S)$ et la confronter aux résultats et conclusions précédemment décrits.

La vitesse de déformation $\dot{\varepsilon}_{\mathrm{s}}$ est donnée par la formule d'Orowan

$$
\dot{\varepsilon}_{\mathrm{s}}=\psi \Phi_{\mathrm{m}} b \bar{V}
$$

dans laquelle $\psi \simeq 0,5$ est un facteur géométrique tenant compte des différents plans activés et de l'orientation des grains, $\Phi_{\mathrm{m}}$ la densité de dislocations mobiles, $b$ le vecteur de Burgers et $\bar{V}$ la vitesse moyenne des dislocations. Nous allons expliciter chaque terme de cette équation.

La densité totale de dislocations est en général une fonction puissance de la contrainte et comme il est admis que $\left(\Phi_{\mathrm{m}} / \Phi_{\mathrm{t}}\right) \simeq$ Cte, on montre que

$$
\Phi_{\mathrm{m}}=K(\sigma / G b)^{m},
$$

relation souvent vérifiée par l'expérience et en particulier avec $m=2$. Compte tenu de l'équation aux dimensions de (13) on a $K=K_{1} b^{m-2}$.

A haute température lorsque le mécanisme séquentiel glissement-montée des défauts linéaires a lieu et que la déformation est due au glissement par exemple, on peut mettre $\bar{V}$ sous la forme

$$
\bar{V}=\left(\frac{L}{h / V_{\mathrm{m}}+L / V_{\mathrm{g}}}\right) .
$$

$V_{\mathrm{m}}$ et $V_{\mathrm{g}}$ sont respectivement les vitesses de montée et de glissement des dislocations, $L$ étant la distance couverte par un défaut dans le plan de glissement lorsque celui de tête est monté d'une hauteur $h$. En combinant (12), (13) et (14) on obtient

$$
\dot{\varepsilon}_{\mathrm{s}}=\frac{K_{1}}{2 b}\left(\frac{\sigma}{G}\right)^{m}\left[\frac{L}{h / V_{\mathrm{m}}+L / V_{\mathrm{g}}}\right] \text {. }
$$

On peut noter que cette formule est générale, en effet, aucune hypothèse sur le mécanisme contrôlant la déformation totale n'a été faite. On obtient une formule similaire si la déformation est due à la montée, le numérateur étant remplacé par $h$.

A ce stade, pour pouvoir expliciter $V_{\mathrm{m}}$ et $V_{\mathrm{g}}$ il est nécessaire de faire des hypothèses d'ordre physique quant aux mécanismes de déformation par superdislocations. A haute température, en accord avec Flinn [69] et Mukherjee et Dorn [26], nous considérons que les superdislocations sont dans un état métastable, c'est-à-dire que si la contrainte appliquée n'est pas suffisante pour créer directement une paroi antiphase, la dislocation considérée ne peut se mouvoir que lorsque des mauvaises liaisons du type AA ou BB se forment en tête de celle-ci, sous l'action combinée de l'agitation thermique et de la contrainte. Il faut noter que cette hypothèse de traînage est conforme au modèle de Brown [3] fondé sur la variation continue de l'ordre à travers la frontière antiphase joignant les partielles d'une superdislocation. D'autre part, compte tenu de l'isotrope relative de la frontière observée dans les $\mathrm{B}_{2}$, cet effet se manifeste aussi bien pour la montée que pour le glissement des superdislocations, indépendamment du temps passé pour glisser ou monter [70], d'où la validité d'une formule du type (14). Le modèle de blocage par anisotropie d'ordre est également conforme à cette idée de traînage. En fait, pour l'ensemble de ces modèles, le déplacement de la dislocation de tête se manifeste toujours par la création de mauvaises liaisons. Ainsi, si l'indice $i$ est égal à $\mathrm{m}$ ou $\mathrm{g}$, selon le mécanisme qui gère la déformation (montée ou glissement), en accord avec Mukherjee et Dorn [26] et à l'aide du formalisme sur la dynamique des dislocations basé sur la thermodynamique des processus réversibles, $V_{i}$ peut s'écrire :

$$
\begin{array}{rl}
V_{i}=b\left(v^{+}-v^{-}\right)=2 & v b Z \exp -\frac{\Delta F}{k T} \times \\
& \times \exp -\frac{e_{i}}{k T} \sinh \frac{A_{i} b \sigma}{k T} ;
\end{array}
$$

$v^{+}$et $v^{-}$sont respectivement les fréquences de saut en avant et en arrière des atomes en tête de la dislocation, $v$ est la fréquence de Debye, $Z$ est l'indice de coordination, $\Delta F$ l'énergie libre d'activation de la diffusion moyenne pour faire progresser la dislocation considérée, $e_{i}$ est l'énergie par aire par atome de la paroi antiphase et $A_{i}$ l'aire par atome de celle-ci.

$A_{i}=a^{2} \sqrt{h^{2}+k^{2}+l^{2}}$ ou $a^{2} / 2 \sqrt{h^{2}+k^{2}+l^{2}}$,

suivant que la somme des indices du plan de mouvement $(h, k, l)$ est impaire ou paire [71], $a$ étant le paramètre du réseau.

En remarquant que, d'une part pour les métaux C.C,

$$
D=v b^{2} \exp -\frac{\Delta F}{k T}=v b^{2} \exp -\frac{\Delta S}{k} \exp -\frac{\Delta H}{k T},
$$

$\Delta S$ et $\Delta H$ étant l'entropie et l'enthalpie d'activation de la diffusion moyenne aléatoire et que d'autre part $A_{i} b \sigma \ll k T,(16)$ se réécrit :

$$
V_{i}=\frac{2 Z A_{i} D \sigma}{k T} \exp -\frac{e_{i}}{k T} .
$$

En écrivant (15) à l'aide de (19), on arrive à la généralisation de la formule de Dorn

$$
\frac{\dot{\varepsilon}_{\mathrm{s}} k T}{D G b}=C \Phi(S)\left(\frac{\sigma}{G}\right)^{n} \text { avec } n=m+1
$$

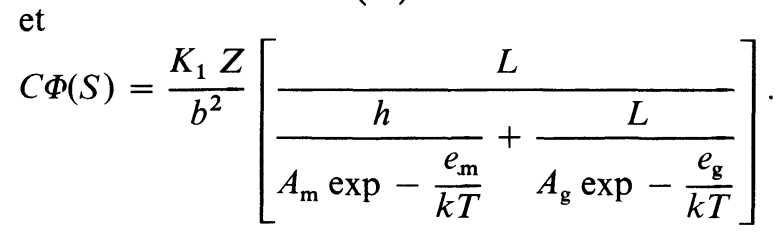


Cette expression appelle à plusieurs remarques d'ordre physique ;

i) L'ordre $S$ n'apparaît dans l'équation (20) que par l'énergie de paroi antiphase $e_{i}$ que nous explicitons ci-dessous.

ii) Cette équation ne fait aucune hypothèse d'ordre énergétique sur l'énergie de paroi antiphase mais il faut voir qu'indépendamment de $n$, la superdislocation est plus lente sur l'une des séquences du mouvement, c'est-à-dire que l'un des termes du dénominateur de l'expression (20) est négligeable devant l'autre. $\Phi(S)$ se réduit à :

$$
\Phi(S)=\exp -\frac{e_{i}(S)}{k T} \quad \text { avec } \quad e_{i}=\Gamma_{i} A_{i}
$$

et $i=\mathrm{m}$ ou $\mathrm{g}$.

$\Gamma_{i}$ est simplement l'énergie de paroi antiphase par unité d'aire. On justifie ainsi la représentation Ln $M=f(1 / T)$ à $(\sigma / G)$ constant, dont la pente est telle que :

$$
\left(\frac{\partial \operatorname{Ln} M}{\partial-\frac{1}{k T}}\right)_{(\sigma / G)}=e_{i}-\frac{1}{k T}\left(\frac{\partial e_{i}}{\partial-\frac{1}{k T}}\right)_{(\sigma / G)} .
$$

Donc, lorsque $e_{i}$ varie peu avec $T$, c'est-à-dire pour $S$ assez grand, on aboutit directement à $e_{i}$.

iii) Si $m=2$, on a $n=3$ et l'on retrouve la formulation de Weertman [43] lorsque le glissement visqueux des dislocations contrôle la déformation,

$$
\left(\frac{\dot{\varepsilon}_{\mathrm{s}} k T}{D G b}\right)=\frac{Z K_{1} A_{\mathrm{g}}}{b^{2}} \exp -\frac{e_{\mathrm{g}}}{k T}\left(\frac{\sigma}{G}\right)^{3}
$$

avec

$$
\Phi(S)=\exp -\frac{e_{\mathrm{g}}}{k T}
$$

Avec $m=3,5$, on a $n=4,5$ et l'on aboutit au modèle de Weertman [72] sur le fluage contrôlé par la montée des dislocations; $\Phi(S)=\exp -\frac{e_{\mathrm{m}}}{k T}$.

Nous explicitons maintenant $e_{i}=\Gamma_{i} A_{i}$. Pour un vecteur de Burgers $\mathbf{b}=\frac{1}{2} a$ [111] commun aux structures du type $B_{2}$ et en accord avec Flinn [69] et Brown et al. [71], on a

$$
\Gamma_{i}=\frac{4 V_{1} h}{a^{2} \sqrt{h^{2}+k^{2}+l^{2}}} \text { avec } \begin{aligned}
& h \geqslant k \geqslant l \\
& h \geqslant k+l
\end{aligned}
$$

ou

$$
\Gamma_{i}=\frac{2 V_{1}(h+k+l)}{a^{2} \sqrt{h^{2}+k^{2}+l^{2}}} \text { avec } \begin{aligned}
& h \geqslant k \geqslant l \\
& h \leqslant k+l
\end{aligned}
$$

pour une paroi conservative et pour $S=1$ en ne tenant compte que des premiers voisins par l'intermédiaire de l'énergie d'ordre $V_{1}$.
On montre alors que

$$
V_{1}=\left(\frac{V_{\mathrm{AA}}+V_{\mathrm{BB}}}{2}\right)-V_{\mathrm{AB}}=\frac{k T_{\mathrm{c}}}{4} .
$$

En faisant intervenir l'ordre, de manière heuristique comme l'ont fait de nombreux auteurs [2], sous la forme $S^{P}$ et compte tenu des $A_{i}(17)$, on aboutit à :

$$
\Phi(S)=\exp -\frac{A\left(h, k, l, V_{1}\right) S^{P}}{k T}
$$

avec

$$
\begin{aligned}
A\left(h, k, l, V_{1}\right) & =k T_{\mathrm{c}} h \\
& =\frac{k T_{\mathrm{c}} h}{2}
\end{aligned}
$$

pour

$$
\begin{aligned}
& (h+k+l) \text { impair } \\
& (h+k+l) \text { pair }
\end{aligned}, \quad\left\{\begin{array}{l}
h \geqslant k \geqslant l \\
h \geqslant k+l
\end{array}\right.
$$

et

$$
\begin{aligned}
A\left(h, k, l, V_{1}\right) & =\frac{k T_{\mathrm{c}}}{2}(h+k+l) \\
& =\frac{k T_{\mathrm{c}}}{4}(h+k+l)
\end{aligned}
$$

pour

$$
\begin{aligned}
& (h+k+l) \text { impair } \\
& (h+k+l) \text { pair }
\end{aligned}, \quad\left\{\begin{array}{l}
h \geqslant k \geqslant l \\
h \leqslant k+l
\end{array} .\right.
$$

On a bien $\Phi(S)=1$ pour $S=0$.

De manière à confronter les valeurs expérimentales des énergies obtenues par la pente des courbes Ln $M=f(1 / T)_{(\sigma / G)}$ sur les différents alliages étudiés, avec l'équation (24) dans laquelle $\Phi(S)$ est conforme à (28), il faut expliciter l'évolution de $S$ avec $T$ ou avec la température réduite $T / T_{\mathrm{c}}$. Ceci ne peut être fait à l'aide des modèles classiques de Bragg-Williams ou de Cowley qui conduisent aux équations transcendantes (9) et (10). Cependant pour $0,85 \leqslant T / T_{\mathrm{c}} \leqslant 1$, lorsque le paramètre d'ordre varie fortement, de nombreux auteurs ont montré que l'on peut écrire

$$
S=B\left(1-\frac{T}{T_{\mathrm{c}}}\right)^{\beta} \quad[42,47,73]
$$

Cette formulation est conforme à la définition thermodynamique générale d'une transformation du second ordre [73]. Les valeurs de $\beta$ et $B$ déduites du modèle d'Ising et qui convergent de manière satisfaisante vers la réalité expérimentale sont $\beta=0,3$ pour $B=1,5[42,47]$. Lorsque $T \rightarrow T_{\mathrm{c}}$, les équations (9) et (10) tendent effectivement vers (29) mais avec des valeurs de $\beta$ différentes.

Comme nous l'avons vérifié dans la première partie de cet article, le modèle de Cowley est celui qui se rapproche le mieux de la réalité puisque dans ce cas, $\beta$ est le plus proche de 0,3 .

Pour $T / T_{\mathrm{c}} \leqslant 0,45$, on a en général $S \simeq 1$. 
Si l'on calcule (24) à l'aide de (28) et (29) on aboutit à :

$$
\begin{aligned}
\left(\frac{\partial \operatorname{Ln} M}{\partial-\frac{1}{k T}}\right)_{(\sigma / G)} & =A\left(h k l, V_{1}\right) B^{P}\left(1-\frac{T}{T_{\mathrm{c}}}\right)^{\beta P}\left[1+\frac{\beta P \frac{T}{T_{\mathrm{c}}}}{1-\frac{T}{T_{\mathrm{c}}}}\right]=A\left(h k l, V_{1}\right) g\left(\frac{T}{T_{\mathrm{c}}}, P\right) \\
& =e_{i}\left[1+\frac{\beta P \frac{T}{T_{\mathrm{c}}}}{1-\frac{T}{T_{\mathrm{c}}}}\right] ;
\end{aligned}
$$

formule valable pour $T / T_{\mathrm{c}} \geqslant 0,8$.

Ainsi, lorsque $T / T_{\mathrm{c}} \rightarrow 1$ c'est-à-dire lorsque $S \rightarrow 0,\left(\frac{\partial \operatorname{Ln} M}{\partial-\frac{1}{k T}}\right)_{\sigma / G} \rightarrow \infty$ et lorsque $T / T_{\mathrm{c}}$ est petit, $S \rightarrow 1,\left(\frac{\partial \operatorname{Ln} M}{\partial-\frac{1}{k T}}\right)_{(\sigma / G)} \rightarrow e_{i}$, ce qui est conforme aux faits expérimentaux. La condition $S \simeq 1$ est obtenue dès $T / T_{\mathrm{c}} \simeq 0,5$.

On peut remarquer que le modèle de Brown [3] sur la dilution de la paroi antiphase conduit pour $T / T_{\mathrm{c}} \geqslant 0,5$ à $S=S_{0}\left(1-\frac{T}{T_{\mathrm{c}}}\right)^{1 / 2}$ et si l'ordre intervient en $S^{P^{\prime}}$, [34] se réécrit :

$$
\left(\frac{\partial \operatorname{Ln} M}{\partial-\frac{1}{k T}}\right)_{(\sigma / G)}=A\left(h k l, V_{1}\right) B^{\prime P^{\prime}}\left(1-\frac{T}{T_{\mathrm{c}}}\right)^{P^{\prime}\left(\beta+\frac{1}{2}\right)}\left[1+\frac{P^{\prime}\left(\beta+\frac{1}{2}\right) \frac{T}{T_{\mathrm{c}}}}{1-\frac{T}{T_{\mathrm{c}}}}\right],
$$

formule identique à (30) si $P^{\prime}=\left(\frac{\beta P}{\beta+\frac{1}{2}}\right)$ et $B^{\prime}=B\left(\frac{\beta+\frac{1}{2}}{\beta}\right)$.

Nous avons représenté sur la figure 11 conformément à $(30), \quad \operatorname{Ln}\left(g\left(T / T_{\mathrm{c}}, P\right)\right)=f\left(T / T_{\mathrm{c}}\right)$ pour $T / T_{\mathrm{c}} \geqslant 0,8, \beta=0,3, B=1,5$ et pour. $P=1$ et 2 . Si $T / T_{\mathrm{c}} \leqslant 0,45, g\left(T / T_{\mathrm{c}}\right)=1$. La valeur $P=2$ a été rapportée de nombreuses fois $[2,3,14,22]$, cependant, le calcul complet de l'énergie de paroi antiphase créée lors du passage de $n$ dislocations montre qu'en toute rigueur cet exposant n'est justifié que pour $0,7 \leqslant S \leqslant 1$, et pour $S<0,7$ on obtient une dépendance beaucoup plus complexe faisant en outre intervenir l'ordre à courte distance [74].

Le fait important est que cette fonction $g\left(T / T_{\mathrm{c}}, P\right)$, croît de 1 pour $T / T_{\mathrm{c}} \simeq 0,5$ à $\infty$ lorsque $T=T_{\mathrm{c}}$. Le modèle de Brown et Lenton [22] prévoit simplement une fonction de Dirac pour $T=T_{\mathrm{c}}$, donc aucun effet d'ordre en dehors du point critique.

Fig. 11. - Variation de $\operatorname{Ln} g\left(T / T_{\mathrm{c}}, P\right)$ en fonction de $T / T_{\mathrm{c}}$. Confrontation avec les résultats expérimentaux obtenus sur les différents alliages pour les plans (111), (110) et (100).

[Variations of $\operatorname{Ln} g\left(T / T_{\mathrm{c}}, P\right)$ versus $T / T_{\mathrm{c}}$. Comparison with the experimental results obtained for the (111), (110) and (100) planes in the different alloys.]

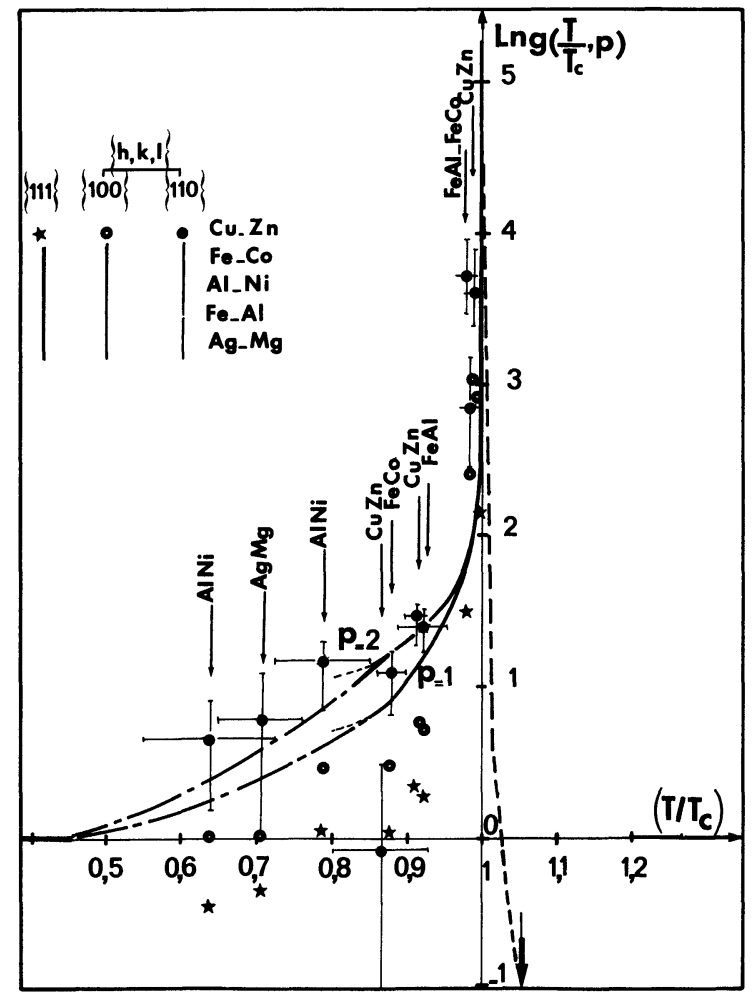


En posant $A=\alpha k T_{\mathrm{c}}$, nous reportons sur cette figure les points expérimentaux $\operatorname{Ln}\left[\frac{\left(\frac{\partial \operatorname{Ln} M}{\partial-1 / k T}\right)_{(\sigma / G)}}{\alpha k T_{\mathrm{c}}}\right]$ obtenus sur les différents alliages pour les plans (110), (100) et (111) avec $\alpha=1 / 2,1$ et $3 / 2$ conformément à l'équation (28). Ces plans sont connus pour être les plans de mouvement les plus probables pour cette structure [74]. Les points obtenus se situent effectivement sur des courbes conformes à $g\left(T / T_{\mathrm{c}}, P\right)$ et pour les plans (110), ils se trouvent sur la courbe $g\left(T / T_{\mathrm{c}}, 2\right)$ et montrent dans ce cas que l'énergie de paroi varie comme $S^{2}$. Ceci est en accord avec les différentes théories citées précédemment. Pour les plans (100) et (111), l'exposant $P$ est beaucoup plus faible et inférieur à l'unité. On peut noter que (110) est un plan de glissement des superdislocations très fréquemment observé dans ces structures [2] et qui leur confère selon (27) une énergie de configuration minimum, du moins lorsque les parois sont conservatives [75]. Ceci est consistant avec le fait que les points expérimentaux se trouvent effectivement sur la courbe en $S^{2}$.

Cependant, il est bien évident que seule une observation directe in situ des plans des superdislocations peut trancher sur le mécanisme qui contrôle la déformation à haute température de ces alliages, mais d'un point de vue rhéologique le traînage des superdislocations suivant un plan entraîne l'existence d'une fonction $\Phi(S) \neq 1$ dont les variations sont très rapides pour $0,8 \leqslant T / T_{\mathrm{c}} \leqslant 1$. Il ne faut pas oublier que la formulation (28) n'est valable qu'à haute température lorsque la diffusion est très rapide et pour des valeurs de $(\sigma / G)$ pas trop élevées.

L'amplitude de la transition entre les états désordonné $(\Phi(S)=1)$ et partiellement ordonné $(\Phi(S) \neq 1)$, est fonction de $T_{\mathrm{c}} / T$ et du plan $(k h l)$ considéré. Ainsi pour $T / T_{\mathrm{c}} \simeq 0,6$, c'est-à-dire lorsque $S$ est proche de l'unité, tout en restant dans le domaine des hautes températures, on obtient respectivement pour les plans (110), (100) et (111) des amplitudes $(\Phi(S))^{-1}$ de l'ordre de 2,3, 5,3 et 6,6. Expérimentalement, on obtient 7 et 4,5 respectivement pour $\mathrm{Cu}-\mathrm{Zn}$ et $\mathrm{Fe}_{3} \mathrm{Al}$ et 7,3 pour $\mathrm{Fe}-\mathrm{CoV}$ pour les plus fortes valeurs de $(\sigma / G)$. L'ordre de grandeur est assez bien respecté. Le modèle de Brown et al. [22] prévoit une transition abrupte de l'ordre de 10 à 15 .

Il reste à expliquer les variations de la contrainte interne avec l'ordre. Dans un mécanisme thermiquement activé, on doit s'attendre à ce que la contrainte interne diminue avec la température, ce qui se vérifie expérimentalement (Figs. 2 et 6). En fait, compte tenu que la restauration devient plus difficile lorsque l'ordre augmente, l'équation de Bailey-Orowan [76] se met sous la forme,

$$
\frac{\mathrm{d} \sigma_{\mathrm{i}}}{\mathrm{d} t}=\theta \sigma_{\mathrm{i}}^{n_{\mathrm{e}}} \frac{\mathrm{d} \varepsilon}{\mathrm{d} t}-\frac{\alpha^{\prime} D}{k T} \sigma_{\mathrm{i}}^{n_{\mathrm{r}}} \Phi(S)
$$

où $\theta$ et $\alpha^{\prime}$ sont des constantes géométriques dépendant des unités et $n_{\mathrm{e}}, n_{\mathrm{r}}$ certains exposants qui tiennent compte des modèles choisis pour l'écrouissage $\left(n_{\mathrm{e}}\right)$ et la restauration $\left(n_{\mathrm{r}}\right)$. Dans tous les cas, $n_{\mathrm{e}} \leqslant 0$ et $n_{\mathrm{r}}>0$. Si l'on considère que le régime stationnaire est atteint lorsque $\frac{\mathrm{d} \sigma_{\mathrm{i}}}{\mathrm{d} t}=0$, on trouve la valeur stationnaire

$$
\sigma_{\mathrm{ist}}^{n_{\mathrm{r}}-n_{\mathrm{e}}}=\frac{\theta \dot{\varepsilon}_{\mathrm{s}} k T}{\alpha^{\prime} D \Phi(S)} .
$$

Si $\theta$ et $\alpha^{\prime}$ sont indépendants de la température, $\sigma_{\text {ist }}$ varie comme $\left(\frac{T}{\Phi(S)}\right)^{n_{\mathrm{r}}-n_{\mathrm{e}}}$, ou plus simplement comme $\left(\frac{1}{\Phi(S)}\right)^{n_{\mathrm{r}}-n_{\mathrm{e}}}$ puisque $\Phi(S)$ varie beaucoup plus vite que $T$.

Cette analyse très simple est qualitativement en accord avec les résultats expérimentaux qui montrent que la contrainte interne s'évanouit avec le degré d'ordre, donc qu'elle est une fonction décroissante de $\Phi(S)$ (Figs. 2 et 6). La contrainte interne mesurée interprète donc en partie la résistance au mouvement des superdislocations, cette résistance étant une fonction croissante de l'ordre. Lorsque ce dernier disparaît, $\sigma_{i}$ est extrêmement faible, fait commun à de nombreuses phases $\beta$ [67].

5. Conclusions. - L'étude du fluage à haute température de ces différents alliages de surstructure $B_{2}$ montre que d'un point de vue rhéologique l'ordre n'intervient dans la généralisation de l'équation de Dorn que par le coefficient de diffusion effectif $\bar{D}$ ou $\tilde{D}$ et surtout par la fonction $\Phi(S)$.

Cette équation s'écrit donc

$$
\frac{\dot{\varepsilon}_{\mathrm{s}} k T}{D G b}=C \Phi(S) f\left(\frac{\sigma}{G}\right) .
$$

Si l'on met $f(\sigma / G)$ sous la forme classique d'une fonction puissance $\alpha(\sigma / G)^{n}, n$ croît avec $(\sigma / G)$ de 3,3 à environ 10 .

A l'aide de considérations simples, on montre que

$$
\Phi(S)=\exp -\frac{A\left(h k l, V_{1}\right) S^{P}}{k T} .
$$

Si l'on confronte les valeurs des énergies calculées à partir des pentes des courbes

$$
M=\operatorname{Ln}\left(\frac{\dot{\varepsilon}_{\mathrm{s}} k T}{D G b}\right)_{(\sigma / G)}=f\left(\frac{1}{T}\right)
$$

et celles déduites du modèle lorsque l'on explicite $\Phi(S)$ en fonction de la température réduite $T / T_{\mathrm{c}}$, on trouve que pour un plan $(h k l)$ donné, l'accord est correct pour une valeur discrète de $P$. Pour (110), plan de minimum d'énergie de configuration pour une paroi conservative, on trouve $P=2$, valeur très souvent rapportée pour la variation de l'énergie de paroi 
antiphase avec le degré d'ordre ; $\Gamma_{i}=\delta k T_{\mathrm{c}} S^{2}$. Pour les plans (100) et (111), $P$ est inférieur à l'unité. Cependant, il est très délicat de raisonner sur les indices, surtout dans le cas de polycristaux où plusieurs systèmes peuvent être activés et il est, par conséquent, bien difficile de conclure si c'est la montée ou le glissement qui contrôle le fluage de ces alliages.

Cependant, d'un point de vue théorique, le traînage des superdislocations selon certains plans entraîne l'existence d'une fonction $\Phi(S)$ qui varie très rapidement avec l'ordre au voisinage du point critique $0,85 \leqslant T / T_{\mathrm{c}} \leqslant 1$. Au-delà, lorsque $S$ est assez grand, tout en restant dans le domaine de validité des hypothèses des hautes températures, $\Phi(S)$ varie assez peu. Ainsi entre les états désordonné et ordonné, $M$ varie continûment et pour $T / T_{\mathrm{c}} \simeq 0,6$, on obtient des amplitudes comprises entre 4 et 7 .

Les contraintes internes mesurées reflètent la résistance au mouvement des superdislocations et varient dans le même sens que le degré d'ordre.

Remerciements. - L'ensemble de ce travail a été effectué à l'aide d'un contrat A.T.P., Rupture et fluage, du C.N.R.S., que nous remercions.

\section{Bibliographie}

[1] Stoloff, N. S. et Davis, R. G., Prog. Mater. Sci. 13 (1966).

[2] Proceedings of the Int. Symp. on Order-Disorder Transformations in Alloys, 3-6 Sept. 1973. Tubingen, Germany (Springer Verlag, Berlin) 1974.

[3] Brown, N., Phil. Mag. 4 (1959) 693

[4] Brown, N., Acta. Met. 7 (1959) 210.

[5] Presnyakov, A. A. et Starikova, G. V., Fiz. Met. Metall. 12 (1969) 873

[6] Vidoz, A. E. et Brown, L. M., Phil. Mag. 7 (1962) 1167.

[7] Druot, P., Lexcellent, C. et Oytana, C., C. R. Hebd. Séan. Acad. Sci. 278 (1974) 1267.

[8] WestbrooK, J. H. et Wood, D. L., Trans. AIME 224 (1962) 1024.

[9] Terry, J. C. et Smallmann, R. E., Phil. Mag. 8 (1964) 1827.

[10] MukherJeE, A. K. et Dorn, J. E., Trans. AIME, 230 (1964) 1065.

[11] Marcinkowski, M. J. et Chessin, H., Phil. Mag. 10 (1964) 837.

[12] Marcinkowski, M. J. et Fisher, R. M., Trans. AIME, 233 (1965) 293.

[13] Dinhut, J. F., Emery, J. P. et Moine, P., Phys. Status Solidi (a) 12 (1972) 153.

[14] Demenet, J. L., Emery, J. P. et Moine, P., Revue Phys. Appl. 9 (1974) 721.

[15] Dinhut, J. F., Bonou, T. et Moine, P., Acta. Met. 24 (1976) 445.

[16] Emery, J. P. et Moine, P., Mém. Sci. Rev. Met. (1976) 257.

[17] Rozner, A. G. et Wasilewski, R. J., J. Inst. Met. 94 (1966) 169.

[18] Lawll., A., Vidoz, E. A. et Cahn, R. W., Actu. Mc'. 9 (1966) 287.

[19] Marcinkowski, M. J., Taylor, M. E. et Kayser, F. X., J. Mat. Sci. 10 (1975) 406.

[20] Herman, M. et Brown, N., Trans. AIME 206 (1956) 604.

[21] Gittins, A. et Gifkins, R. C., Acta. Met. 16 (1968) 81.

[22] Brown, N. et Lenton, D. R., Acta. Met. 17 (1969) 669.

[23] Oytana, C., Mermet, A. et Druot, P., Mat. Sci. Eng. 14 (1974) 89.

[24] Delobelle, P. et Oytana, C., Mém. Sci. Rev. Met. 73 (1976) 293.

[25] Delobelle, P., Druot, P., Mermet, A. et Oytana, C., Revue Phys. Appl. 13 (1978) 867.

[26] MukherJeE, A. K. et Dorn, J. E., J. Inst. Met. 93 (1965) 397.

[27] Hren, J. A. et Sherby, O. D., Appl. Mat. Res. 4 (1965) 41.

[28] Delobelle, P., Mermet, A. et Oytana, C., Mat. Sci. Eng. 41 (1979) 203

[29] Vandervoort, R. R., Mukherjee, A. K. et Dorn, J. E., Trans. AIME 59 (1966) 930

[30] Lawley, A., Coll, J. A. et CahN, R. W., Trans. AIME 218 (1960) 166.

[31] Surova, E. A., Bystrov, L. N. et Ivanov, L. I., Izvest. Akad. Nauk. SSSR 4 (1963) 99.
[32] Abramyan, E. A., Ivanov, L. I., Izvest. Akad. Nauk. SSSR 6 (1966) 110.

[33] Mukherjee, A. K., Bird, J. E. et Dorn, J. E., Trans. AIME, 62 (1969) 155

[34] Ahlquist, C. N. et Nix, W. D., Scripta Met. 3 (1969) 679.

[35] LeXcellent, C., Thèse Besançon, France (1977).

[36] Brebec, G. et Poirier, J. P., Acta. Met. 23 (1975) 125.

[37] Chin, B. A., Pound, G. M. et Nix, W. D., Met. Trans. 8A (1977) 1517.

[38] Weertman, J., Trans. ASME 61 (1968) 681

[39] Burton, B. et Bastow, B. D., Acta. Met. 21 (1973) 13.

[40] Kuper, B., Lazarus, D., Manning, R. et Tomizuka, T., Phys. Rev. 104 (1956) 1536.

[41] Ugaste, Yu. E. et Pimenov, V. N., Phys. Met. Metall. 31 (1971) 140.

[42] Rathmann, O. et Als-Nielsen, J., J. Phys. Rev. 9 (1974) 3921.

[43] Weertman, J., J. Appl. Phys. 28 (1957) 1185.

[44] Gupta, D. et Lieberman, D. S. dans "Ordered Alloys" B. K. Kear et C. T. Sims Edit. Clayton's Baton Rouge (1970) 195.

[45] Oytana, C., Delobelle, P. et Mermet, A., J. Mat. Sci. 14 (1979) 549.

[46] Funamizu, Y. et Watanabe, K., J. Japan. Inst. Met. 39 (1975) 1087.

[47] Walker, C. B. et Chipman, D. R., Phys. Rev. 4 (1971) 3104.

[48] Dietrich, O. W. et Nielsen, J., The Danish Atomic Energy Report from Research Etablishment at Riso. Denmark (1967).

[49] Hirone, T., Kunitomi, N. et Sakamoto, M., J. Phys. Soc. Jap. 13 (1958) 840.

[50] Fishman, S. G., Gupta, D. et Lieberman, D. S., Phys. Rev. 2 (1970) 1451.

[51] Hirano, K. et Cohen, M., Trans. Jap. Inst. Met. 13 (1972) 96.

[52] Hirano, K., Ijima, Y., Araki, K. et Homma, H., Trans. Iron. Stell. Inst. Jap. 17 (1977) 194.

[53] Chang, Y. A., Himmel, L. et Neumann, J. P., J. Appl. Phys. 38 (1967) 649.

[54] Hagel, W. C. et WestbrooK, J. H., Trans. AIME 221 (1961) 951.

[55] Domian, H. A. et Aaronson, H. I., "Int. Conf. on Diffusion in BCC Metals » Am. Soc. Met., Gatlinburg, Tennessee (1974) 209.

[56] Robinson, P., Intermetallic Compounds, J. H. Westbrook edit. (John Wiley and Sons, New York) Chap. III (1967) 38.

[57] RimlingeR, L., Thèse d'état Nancy, France (1970).

[58] Hren, J. A., Phys. Status Solidi (a) 3 (1963) 1603.

[59] GröBNer, P., Hutnické Listy 10 (1955) 200.

[60] Hirano, K. et Hishinuma, A., J. Jap. Inst. Met. 32 (1968) 516. 
[61] Larikov, L. N., Fal'chenko, V. M., Polishchuk, D. F., Ryabov, V. R. et Lozovskaya, A. V., Zesh. Pokr. Na. Metall. 3 (1970) 91.

[62] LaRikov, L. N., Autom. Svarka 24 (1971) 71.

[63] Khachaturyan, Progress in Materials Science (Pergamon Press) 22 (1978) 88.

[64] Wasilewski, R. J., Trans. AIME 236 (1966) 455.

[65] Shankar, S. et Seigle, L. L., Met. Trans. 91 (1978) 167.

[66] Berkowitz, A. E., Jaumot, F. E. et Nix, W. D., Phys. Rev. 95 (1954) 1185.

[67] Bornier, L., Delobelle, P., Mermet, A. et Oytana, C., Met. Trans. 10A (1979) 1944.
[68] Garofalo, F., Déformation et rupture par fluage (Dunod, Paris) 1970.

[69] Flinn, P. A., Trans. AIME 218 (1960) 145.

[70] Brown, N., Scripta Met. 4 (1970) 417.

[71] CupshalK, S. G. et Brown, N., Acta Met. 16 (1968) 657.

[72] Weertman, J., J. Appl. Phys. 28 (1957) 362.

[73] Corey, C. L., Ordered Alloys, B. K. Kear, CT Sims. Clayton's (Baton Rouge) 1970, 241.

[74] Eymery, J. P., Garem, H. et Moine, P., Phys. Status Solidi (a) 14 (1972) 431.

[75] Mitchell, J. B., Scripta Met. 4 (1970) 411.

[76] Bailey, R. W., J. Inst. Metals 35 (1926) 27. 